RACAR : Revue d'art canadienne

Canadian Art Review

RACAR

\title{
Les Jeux d'Enfants, tapisseries italiennes et flamandes pour les Gonzague
}

\author{
Guy Delmarcel et Clifford M. Brown
}

Volume 15, numéro 2, 1988

URI : https://id.erudit.org/iderudit/1073371ar

DOI : https://doi.org/10.7202/1073371ar

Aller au sommaire du numéro

Éditeur(s)

UAAC-AAUC (University Art Association of Canada | Association d'art des universités du Canada)

ISSN

0315-9906 (imprimé)

1918-4778 (numérique)

Découvrir la revue

Citer cet article

Delmarcel, G. \& Brown, C. M. (1988). Les Jeux d'Enfants, tapisseries italiennes et flamandes pour les Gonzague. RACAR : Revue d'art canadienne / Canadian Art Review, 15(2), 109-121. https://doi.org/10.7202/1073371ar
Résumé de l'article

Two sets of tapestries depicting Puttini can be related to the patronage of the Gonzaga family. On his death in 1563, Cardinal Ercole Gonzaga possessed a series of 15 tapestries with Puttini designed by Giulio Romano and woven in the workshop of Nicholas Karcher, probably during his stay in Mantua between 1539 and 1545. The iconography of the four complete surviving panels relates only casually to the description of the puttini in Philostratos's Images. As the patron of the set, Cardinal Ercole might have wished an emblematic ambiguity for these scenes with reference both to pagan Dionysiac life and to the Christian eucharistic theme of wine. Accordingly, the so-called Bark of Venus might be interpreted in several ways. Ercole's inventory of 1563 also mentions a set of twelve spallere or dorsal tapestries with "Fortune led by four Eagles." Giulio Romano's design in Haarlem, where the woman holding a rudder may stand for Fortuna, can be related to these now lost weavings. As the woman in the Bark of Venus also holds a rudder, she probably alludes to Fortuna leading the good fame of Ercole whose coat of arms appears on the top of the weaving and whose name is written by a genius on a cartellino on the foreground. Moreover, as the general scheme of this tapestry is closely related to that of the Miraculous Draught of Fishes in Raphael's Acts of the Apostles set, the Bark of Venus/Fortune might be presumed to have an underlying Christian meaning.

Ercole's younger brother, Ferrante, founder of the Guastalla branch of the Gonzaga family, ordered a similar set of Puttini from the Brussels workshop of Willem de Pannemaker. This can be deduced from correspondence with Cardinal Granvella in 1571-74 concerning a copy of these tapestries from the same weaver. When the painter Giovanni Battista Lodi proposed in February 1552 to entrust Ferrante's order for tapestries woven with gold and silver to Willem de Pannemaker, he was unquestionably referring to the Marzotto Puttini, the only series with precious metal in the Gonzaga collection, where it can be traced until 1709. Thereafter, the set can be documented in various Venetian collections until it was acquired by its present owner, the Marzotto family in Trissino.

The copies made for Granvella, partially preserved in the Spanish Royal Collection, are identical to the six Marzotto tapestries. Both sets bear the mark of Pannemaker, but the Marzotto tapestries made for Ferrante are the first edition.

The Brussels Puttini set relies on Northern Italian models, either from the workshop of Giulio Romano or from Milanese painters. Unlike the set created for Cardinal Ercole, many details made it clear that the cartoons created for Ferrante were designed in Flanders. The six pieces, representing the harvest of the grapes, the pressing of the wine, and the dance are thus devoted to the theme of Dionysiac paradise and peace as a dialectical complement to Ferrante's older Brussels set of the Fructus Belli. The building in the right background of the Dance probably represents, at least in an idealized way, the Villa Gualtiera-Simonetta, Ferrante's residence in Milan.
Tous droits réservés (C) UAAC-AAUC (University Art Association of Canada | Association d'art des universités du Canada), 1988
Ce document est protégé par la loi sur le droit d'auteur. L’utilisation des services d'Érudit (y compris la reproduction) est assujettie à sa politique d'utilisation que vous pouvez consulter en ligne.

https://apropos.erudit.org/fr/usagers/politique-dutilisation/ 


\section{Les Jeux d'Enfants, tapisseries italiennes et flamandes pour les Gonzague}

GUY DELMARCEL

Musées royaux d'Art et d'Histoire, Bruxelles

et

CLIFFORD M. BROWN

Carleton University

Two sets of tapestries depicting Puttini can be related to the patronage of the Gonzaga family. On his death in 1563, Cardinal Ercole Gonzaga possessed a series of 15 tapestries with Puttini designed by Giulio Romano and woven in the workshop of Nicholas Karcher, probably during his stay in Mantua between 1539 and 1545. The iconography of the four complete surviving panels relates only casually to the description of the puttini in Philostratos's Images. As the patron of the set, Cardinal Ercole might have wished an emblematic ambiguity for these scenes with reference both to pagan Dionysiac life and to the Christian eucharistic theme of wine. Accordingly, the so-called Bark of Venus might be interpreted in several ways. Ercole's inventory of 1563 also mentions a set of twelve spallere or dorsal tapestries with "Fortune led by four Eagles." Giulio Romano's design in Haarlem, where the woman holding a rudder may stand for Fortuna, can be related to these now lost weavings. As the woman in the Bark of Venus also holds a rudder, she probably alludes to Fortuna leading the good fame of Ercole whose coat of arms appears on the top of the weaving and whose name is written by a genius on a cartellino on the foreground. Moreover, as the general scheme of this tapestry is closely related to that of the Miraculous Draught of Fishes in Raphael's Acts of the Apostles set, the Bark of Venus/Fortune might be presumed to have an underlying Christian meaning.

Ercole's younger brother, Ferrante, founder of the Guastalla branch of the Gonzaga family, ordered a similar set of Puttini from the Brussels workshop of Willem de Pannemaker. This can be deduced from correspondence with Cardinal Granvella in 1571-74 concerning a copy of these tapestries from the same weaver. When the painter Giovanni Battista Lodi proposed in February 1552 to entrust Ferrante's order for tapestries woven with gold and silver to Willem de Pannemaker, he was unquestionably referring to the Marzotto Puttini, the only series with precious metal in the Gonzaga collection, where it can be traced until 1709. Thereafter, the set can be documented in various Venetian collections until it was acquired by its present owner, the Marzotto family in Trissino.

The copies made for Granvella, partially preserved in the Spanish Royal Collection, are identical to the six Marzotto tapestries. Both sets bear the mark of Pannemaker, but the Marzotto tapestries made for Ferrante are the first edition.

The Brussels Puttini set relies on Northern Italian models, either from the workshop of Giulio Romano or from Milanese painters. Unlike the set created for Cardinal Ercole, many details made it clear that the cartoons created for Ferrante were designed in Flanders. The six pieces, representing the harvest of the grapes, the pressing of the wine, and the dance are thus devoted to the theme of Dionysiac paradise and peace as a dialectical complement to Ferrante's older Brussels set of the Fructus Belli. The building in the right background of the Dance probably represents, at least in an idealized way, the Villa Gualtiera-Simonetta, Ferrante's residence in Milan.
Les Gonzague, seigneurs de Mantoue et de nombreux autres domaines en Italie du Nord, furent des collectionneurs avertis et enthousiastes de tapisseries, surtout pendant la Renaissance. Deux 
tentures vouées à un même thème, celui des Jeux d'Enfants, peuvent illustrer un aspect de leur activité en faveur des arts textiles ${ }^{1}$.

Parmi d'autres thèmes profanes, le sujet des Jeux d'Enfants était déjà bien connu et répandu dans la tapisserie dès le $x^{e}$ siècle. Ainsi, la comtesse Marguerite de Flandre, épouse de Philippe le Hardi, duc de Bourgogne, laissa dans son héritage, en 1405, " un pavillon de haulte liche de soie à champ vermeil ouvré et semé de petits enfants faisant esbatement ", et son petit-fils, Philippe le Bon, possédait lors de son accession au pouvoir en 1430 " une riche chambre [...] d'arbres et herbaiges et petiz enfans ". Citons aussi " la chambre aux petits enfants " présente au château de Nérac lors du décès d'Anne d'Armagnac en $1472^{2}$. Toutes ces tentures ont disparu. Celles qui existent encore datent du $\mathrm{xvI}^{\mathrm{e}}$ siècle, et elles peuvent être mises toutes en relation avec le mécénat des Gonzague. Examinons ici quelques aspects historiques et iconographiques d'une tenture italienne aux armes du cardinal Ercole, et d'une tenture bruxelloise apparentée qui fut commandée par son frère cadet, Ferrante, fondateur de la branche cadette des Gonzague de Guastalla.

\section{LES PUTTINI DU CARDINAL ERCOLE GONZAGA}

La tenture des Puttini ou Jeux d'Enfants ayant appartenu au cardinal Ercole Gonzaga est considérée à juste titre comme un des ensembles textiles les plus prestigieux de la Renaissance italienne (Figs 79 à 84 ). Sa première mention remonte au testament du cardinal, établi le 2 mars 1563, par

1 Cet article faisant partie de notre étude plus large en préparation Flemish and Italian Renaissance Tapestries from the Collections of Ercole and Ferrante Gonzaga, nous renvoyons le lecteur à cette publication future pour les citations d'archives et pour les transcriptions de tous les documents mentionnés ici. En attendant, les références d'archives sont inclues entre parenthèses dans le texte. ASMn $=$ Archivio di Stato di Mantova; ASPr = Archivio di Stato di Parma (Archivio di Gonzaga di Guastalla); BMG = Biblioteca Maldottiana, Guastalla. Bien que de nombreux documents cités dans cet article ne soient pas encore publiés, ceux se rapportant à la collection du cardinal Ercole Gonzaga ont été commentés par Alia Englen, "Gli arazzi di Raffaello: la seria mantovana ", cat. expos. Aspetti dell'arte a Roma prima e dopo Raffaello (Rome, Palazzo Venezia, 1984), 144-45. Nous remercions Candace Adelson, Nello Forti Grazzini, AnnaMaria Lorenzoni, Maria Fernanda Passos Leite, Bert W. Meijer, Francesco Pertegato, Aurora Scotti et surtout le comte Giannino Marzotto, Villa Trissino à Trissino, pour les informations fournies en faveur de cet article

$2 \mathrm{Ch}$. Dehaisnes, Documents et extraits divers concernant l'histoire de l'art dans la Flandre, l'Artois et le Hainaut avant le $\mathrm{XV}^{\prime \prime}$ siècle (Lille, 1886), 343; L. de Laborde, Les ducs de Bourgogne, II (Paris, 1849-1852), 268, no 4262; Ch. Rahlenbeck, "Les tapisseries des rois de Navarre ", Messager des Sciences historiques (1868), 374 et 379 . lequel il lègue à son neveu, le duc de Mantoue « et similmente le tapezzarie nominate delli Puttini per servirsene come meglio parerà a Sua Eccellenza " (ASmn, Busta 333, cc. 267 ff.) ${ }^{3}$. Dans l'inventaire dressé après la mort d'Ercole, survenue le 8 mars 1563 à Trente, la tenture est citée en première place: "tapezzaria di putini, pezzi diece di lana, seta, oro et argento, sopraportere della medema sorte dui, portiere tre di lana, seta et oro, fatto a foglio di lauro con l'arma di Monsignor illustrissimo, in meggio » (ASMn, Registrazioni notarili, 1564, cc. 233 r.). De cet ensemble initial de quinze pièces, ayant compris dix tapisseries figurées, deux dessus de porte et trois portières, pourvues des armes du cardinal dans des feuilles de laurier, quatre tapisseries entières aux armes du cardinal et deux fragments existent encore à la Fondation Gulbenkian à Lisbonne, et un fragment central armorié d'une pièce initialement plus grande au Museo Poldi-Pezzoli à Milan 4 .

Ercole Gonzaga étant devenu cardinal en 1527, on peut dater d'emblée ces pièces entre cette année et 1563, mais la plupart des spécialistes s'accordent d'en attribuer l'exécution par l'atelier de Nicolas Karcher vers 1540, selon des maquettes de Giulio Romano. La paternité de ce dernier est prouvée par plusieurs dessins préparatoires pour cette tenture, et la haute qualité du tissage est comparable à celle des premières tapisseries que Nicolas Karcher tissa à Florence en 1545-1546.

Faute de documents d'archives péremptoires, le doute subsiste à propos du lieu de tissage des Puttini: Ferrare ou Mantoue? Le licier bruxellois Nicolas Karcher fut au service des Este à Ferrare probablement depuis 1517. Le plus ancien document témoignant de la présence de Karcher et de onze personnes de son entourage à Mantoue, un privilège ducal du 8 octobre 1539 , prétend que le duc Federico l'a appelé dans ses terres pour y tisser des tapisseries " secondo gli disegni che gli faremo dare $»^{5}$. Son transfert à Mantoue semble donc

3 W. Braghirolli, Sulle Manifalture di Arazzi in Mantova. Notizie Storiche (Mantoue, 1879), 45.

$4 \mathrm{M}$. Viale Ferrero, Arazzi italiani del Cinquecento, $2^{\mathrm{C}}$ éd (Milan, 1963), 21, 54-55; G. N. Riso Guerreiro, "Some European 'Tapestries in the Calouste Gulbenkian Collection in Lisbon", The Connowseur (April 1970), 229-34; N. Forti Grazzini, Arazzi a Ferrara (Milan, 1982), 76-79 et 161-87; M. Viale Ferrero, "Arazzi", dans Museo PoldiPezzoli. Arazzi-Tappeti-Tessuti copti-Pizzi-Ricami-Ventagli (Milan, 1984), 17-18. Des exemplaires de réédition, sans fils précieux, sont conservés au Victoria and Albert Museum, Londres, et chez. le Marquis de Northampton à Compton Wynyates, cf. G. Wingfield Digby et W. Hefford, Victoria and Albert Museum. The Tapestry Collection: Medieval and Renaissance (Londres, 1980), 67-69.

5 ASmn, Libri dei Decreti, xi.I, c. 56. A. Luzio, Gli arazzi dei Gonzaga restituti dall'Austria (Bergamo, 1919), 34; Forti, Ferrara, 61 . 
avoir été motivé par une commande particulière, pour laquelle des modèles étaient prévus. Or, la tenture des Jeux d'Enfants est la seule encore existante que l'on puisse situer dans cette période. Karcher travaillant déjà à Mantoue avant la mort de Federico, survenue le 28 juin 1540, il n'est donc pas impossible que la tenture fut commencée pour ce dernier. Dès la disparition de Federico, le cardinal Ercole devint régent de Mantoue, en même temps que son frère cadet Ferrante. Tant le tapissier Karcher que l'architecte et peintre Giulio Romano restèrent à Mantoue au service du cardinal : Karcher jusqu'en octobre de 1545, quand il alla travailler pour le grand-duc Cosimo de'Medici à Florence $^{6}$, et Giulio jusqu'à sa propre mort, le 11 november 1546 . Il nous semble dès lors acceptable que la tenture des Jeux d'Enfants peut avoir été tissée à Mantoue entre 1539 et 1545 , durant la présence conjointe de Karcher et de Romano en cette ville ${ }^{7}$. Elle peut avoir été conçue pour le duc Federico et "récupérée " ensuite par le cardinal Ercole. Sur les quatre pièces de Lisbonne, ses armoiries semblent avoir été insérées par renchaînage, ce qui indiquerait qu'elles n'étaient pas prévues sur les métiers dès le début (Fig. 83). De même, le cardinal peut avoir décidé d'ajouter ou de modifier des sujets entiers de tapisseries.

On décrit les quatre tapisseries complètes, à présent à Lisbonne, traditionnellement comme la Barque de Vénus (Fig. 79), le Jeu de Balle (Fig. 80), la Danse (Fig. 81) et la Pêche (Fig. 82) ${ }^{8}$. Pour autant que l'on puisse juger du programme de dix pièces dont il n'en reste que quatre, il est indéniable que ces tissus contiennent plusieurs éléments de la source iconographique antique la plus évidente de ce thème, les Eikones ou Images de Philostrate l'Ancien. On y reconnaît ainsi la cueillette des pommes, le jeu de deux amours avec une pomme (le soi-disant jeu de balle), le carquois de flèches pendu à l'arbre ${ }^{9}$. La description de Philostrate devait être bien connue chez les Gonzague à cette époque. La première traduction du texte grec fut

6 C. Adelson, "Cosimo I de'Medici and the Foundation of Tapestry Production in Florence ", dans Firenze e la Toscana dei Medici nell'Europa del '500 (Florence, 1983), iII, 907.

7 Un tissage durant le deuxième séjour de Nicolas Karcher à Mantoue, de 1555 à 1562, est hautement improbable, et n'est accepté par aucun spécialiste. Nous remercions ici Candace Adelson, qui a bien voulu confirmer notre opinion à propos de la date du tissage des Puttini et qui, elle aussi, envisage une conception initiale de cette tenture pour le duc Federico (lettre de C.A. à G.D., 19 janvier 1988).

8 Ces titres ne se trouvent dans la littérature d'art que depuis Alfred Darcel, "Le Moyen Age et la Renaissance au Trocadéro ", Gazette des Beaux-Arts, xx (1878), 1007. Il n'est pas possible de donner un titre générique aux deux fragments de Lisbonne et à celui de Milan, leurs sujets principaux n'étant plus évidents à cause de leur mutilation.

9 Philostratus the Elder, Imagines, I, 6 (Londres, 1960), 20-29. exécutée en 1510 pour Isabella d'Este, la mère de Federico et d'Ercole, et la plus célèbre représentation artistique de ce fameux chapitre des erotes est la Fête de Vénus, peinte par le Titien en 15181519 pour le "camerino d'alabastro " d'Alfonso d'Este, duc de Ferrare et frère d'Isabella ${ }^{10}$.

D'autre part, on note aussi plusieurs différences par rapport au texte antique. Ainsi, les amours ne cueillent pas seulement des pommes, mais surtout des grappes de raisins; ils s'occupent de la pêche; ils mènent une danse, pendant laquelle l'un d'eux est blessé d'une flèche tombant d'un carquois; la danse des amours est accompagnée par un musicien jouant de la lyre; enfin, Vénus n'est pas représentée sous un rocher près d'une fontaine ou d'une cascade, comme le décrit Philostrate, mais elle vogue en barque sous une tonnelle. Dès lors, la plupart des auteurs s'accordent à voir dans cette tenture une interprétation plus libre, un peu selon la fantaisie de Giulio Romano, qui créa des variations infinies contenues dans l'unité spatiale remarquable obtenue par le cadre de la pergola ${ }^{11}$.

Mercedes Viale Ferrero fut la première à suggérer une polysémie pour cette série. Considérant que le thème des Puttini est une cage vide permettant d'y verser les significations les plus diverses, elle pense que Giulio a doté ces tapisseries d'un programme spécifique et ambigu à la fois. Le sens avant tout compliqué et élusif de la tenture pourrait bien, selon elle, être expliqué comme une allégorie chrétienne, à l'instar des commentaires ultérieurs de Ruscelli à propos de l'imprese du cardinal Ercole. En ce cas, Vénus serait la Vénus céleste ou la Charité, les putti les âmes fidèles, etc. ${ }^{12}$. Cette hypothèse d'interprétation, bien que proposée sans une analyse plus explicite ou fondée par des textes, est très suggestive. Etant également convaincus que cette tenture est plus qu'une fantaisie à l'antique et qu'elle a dû avoir eu un sens bien précis pour son commanditaire (ou ses commanditaires), nous voudrions faire quelques suggestions complémentaires à ce propos, en nous basant sur divers éléments tant historiques que formels.

Notons d'abord que le cardinal Ercole, bien qu'il ait mené une vie amoureuse privée comme tout prélat de la Renaissance, voulait avant tout passer pour un homme d'église pieux. Ainsi, durant sa régence de Mantoue, il ne confiera à Giulio

10 Madrid, Museo del Prado. Cf. la synthèse récente de G. Cavalli-Björkman, "Camerino d'alabastro, a Renaissance Room in Ferrara ", dans cat. expos. The Bacchanals by Titian and Rubens, Nationalmuseum Bulletin, XI, 2 (Stockholm, 1987), 75 et 78 .

11 M. Viale Ferrero, Arazzi italiani (Milan, 1961), 25; Viale, Arazzi Cinquecento, 21; Forti, Ferrara, 76.

12 M. Viale Ferrero, "Arazzo e pittura ", dans Storia dell'arte italiana, ed. P. Fossati (Turin, 1982), III-4, 133-34. 
que des travaux d'architecture religieuse ${ }^{13}$. En plus, des documents signalés par A. Englen prouvent que le cardinal était très sensible aux sujets achetés par lui en tapisserie. Dans une lettre datée du 2 octobre 1557 et adressée au comte Federico Maffei, résidant à Bruxelles, Ercole demanda de lui trouver quelque tenture à sujet religieux : elle devait être "di bellissima sorte " contenant de la soie et un peu d'or et d'argent, " et che insieme fossero di cosa conveniente al grado e profession mia, cioé che non contenessero favola o cosa lasciva, ma qualche historia della scrittura et del Testamento Vecchio" (ASmn, Busta 6512, cc. 79 v. -80 r. $)^{14}$. L'inventaire des tapisseries d'Ercole, dressé après sa mort en 1563, confirme cette propension aux sujets religieux. Des treize tentures mentionnées, cinq sont chrétiennes (Actes des Apôtres, Tobie, Saül, David et Joseph), une est historique (Histoire de Troie), deux sont des Verdures ("fogliami grandi a verdura"), deux ne sont pas explicitées, et trois seulement sont profanes : les "puttini ", une tapisserie " con una fontana et pastori ", et douze "spalere " ou dosserets en laine "della Fortuna condota da quatro Aquile " (ASMn, Registrazioni notarili, 1564, cc. 233).

C'est cette dernière tenture qui peut nous donner une clef possible pour une nouvelle lecture des Puttini. En effet, ces dosserets qui, selon l'inventaire de l'héritier d'Ercole, son neveu le cardinal Federico Gonzaga, étaient "di Fiandra " (ASMn, Busta 333, cc. $290 \mathrm{ff}$ : 7 mars 1566) et qui furent encore employés en 1584 pour orner le palais ducal lors du mariage de Vicenzo Gonzaga et Eléonore de Médicis (ASMn, Busta 204, c. 194) étaient très certainement des panneaux héraldiques pour lesquels Giulio Romano a fourni les maquettes. La description de l'inventaire de 1563 correspond parfaitement à un dessin de Giulio, conservé à la Fondation Teyler de Haarlem: tandis que deux génies tiennent les armes et le chapeau cardinalice d'Ercole, le chariot de l'Etat est tiré par les quatre aigles héraldiques des Gonzague, et mené par une femme tenant un gouvernail de bateau, attribut de la Fortune marine employé fréquemment pendant la Renaissance italienne (Fig. 85) ${ }^{15}$. La devise :

13 F. Hartt, Giulio Romano (New Haven, 1958), 1, 234. Pour une évaluation des activités du cardinal à cette époque, cf. le rapport de Bernardo Navagero à la république de Venise en 1540, dans A. Segarizzi, Relazioni degli ambasciatori veneti al Senato, 4 vols. (Bari, 1912-1919), I, 53-56.

14 Texte déjà cité par A. Englen, " Gli arazzi », 145, note 26.

15 Hartt, Romano, II, fig. 521. Jane T. Martineau décrit ce dessin correctement dans cat. expos. Splendours of the Gonzaga (Londres, 1981-1982), 204, $\mathrm{n}^{\circ} 204$; C. van Tuyll, dans cat. expos. Disegni italiani del Teylers Museum Haarlem provenienti dalle collezioni di Cristina di Svezia e dei principi Odescalchi (Florence et Rome, 1983-1984), 154-55, nº66, confirme
RENOVABITVR VT AQVILAE IVVENTVS ("La jeunesse sera renouvelée comme les aigles"), extraite du Psaume 103 et se rapportant à un texte faisant allusion à la justice du Seigneur, est applicable à la régence du cardinal dès 1540 . Fait intéressant, ce dessin de Giulio, sans aucun doute le petit patron pour ces douze "spallere "flamandes, est considéré en général comme une variante d'un autre modèle pour tapisserie de Giulio, à présent à Chatsworth, et où le chariot est mené par un amour ailé au lieu de la Fortune au gouvernail ${ }^{16}$. Les deux esquisses préparatoires pour ces tapisseries armoriées situent en outre le sujet sous une tonnelle formée par une vigne grimpant le long de troncs d'arbres. Giulio proposait donc ici à son commanditaire une tenture héraldique, où les Puttini varient avec la Fortune pour guider sous une vigne le char de l'Etat des Gonzague, symbolisés par leurs armoires.

Il nous semble que ces mêmes thèmes ont été combinés en partie sur la tapisserie de la Barque de Vénus de la tenture des Jeux d'Enfants, créée elle aussi par Giulio pour les Gonzague (Fig. 79). Les anciens inventaires ne donnent pas de noms détaillés à chaque tapisserie, et les définitions actuelles ne remontent qu'au $\mathrm{XIx}^{\mathrm{e}}$ siècle. Une telle représentation de la barque de Vénus n'est ni fréquente ni évidente durant la Renaissance. Ni Philostrate ni d'autres textes ne décrivent une telle scène. Sur des reliefs de sarcophages antiques, Vénus se transporte sur les flots dans une coquille, et elle y est parfois accompagnée de quelques amours. L'image fit fortune au Quattrocento par le célèbre tableau de Botticelli17

La barque de Vénus, quant à elle, apparaît pour la première fois précisément dans l'art de la tapisserie flamande d'après des modèles italiens. En 1520, peu avant sa mort, le Pape Léon x commanda au célèbre lissier bruxellois Pierre van Aelst, qui avait déjà exécuté les Actes des Apôtres selon Raphaël, une tenture de Grotesques avec Divinités d'après des maquettes de Giovanni da Udine. Cette tenture papale a disparu par la suite, mais elle est connue indirectement par des rééditions et adaptations ultérieures, dues à Perino del Vaga et Amigo Aspertini ${ }^{18}$. Or, la tapisserie de

cette identification et date les deux feuilles vers 1540-1546. Pour le gouvernail comme attribut de la Fortune, cf. G. de Tervarent, Attributs et symboles dans l'art profane de la Renaissance (Genève, 1958), I, 203 et A. Cavallaro, "Pinturicchio a Roma. Il soffitto dei "semidei" nel palazzo di Domenico della Rovere ", Storia dell'Arte, Lx (1987), 158-59.

16 Hartt, Romano, II, fig. 518.

17 E. Wind, Pagan Mysteries in the Renaissance, $2^{e}$ ed. (Harmondsworth, 1967), 128-40 (à propos de Botticelli) et 263-64; A. Rumpf, Die Meerwesen auf den antiken Sarkophag. reliefs (Rome, 1969), 36-39 et pl. 36-37.

18 A. M. L. E. Erkelens, "Rafaëleske Grotesken op enige 
Vénus est décrite dans l'inventaire de Paul irr en 1544 comme " uno panetto con la nave et trionfo de Venere ", et cette composition nous est parvenue par une réédition bruxelloise de vers 1570 et par une copie des Gobelins de 1684-1695. Vénus y trône au milieu d'un grand navire chargé d'amours ailés et entouré d'autres génies chevauchant des monstres marins dans les flots ${ }^{19}$.

Si Giulio Romano peut s'être donc inspiré d'une tenture conçue par un de ses collègues dans la bottegha de Raphaël et exécutée à Bruxelles dès 1520 , il faut toutefois remarquer que Vénus est représentée tout aussi souvent sans la barque, tant sur les variantes des Grotesques de Léon x que sur d'autres tentures bruxelloises de l'époque ${ }^{20}$. Sur la tapisserie du cardinal Ercole, la Vénus marine tient le gouvernail de la barque remplie d'amours, et cette image nous ramène aux dosserets de la Fortune créés par Giulio pour le même cardinal Ercole. Depuis la littérature et l'art de la basse antiquité, la Fortune était associée fréquemment à l'instabilité et aux dangers de la mer, et dès lors pourvue du gouvernail ${ }^{21}$. Le Quattrocento reprend cette image de la Fortune marine, dotée en outre du navire et de la voile, qu'elle tient en voguant sur une boule ou sur une coquille ${ }^{22}$. Cette

Brusselse wandtapijtseries ", Bulletin van het Rijksmuseum, $\mathbf{x}$ (1962), 115-38; P. Boccardo, dans cat. expos. Raffaello e la Cultura raffaellesca in Liguria (Gènes, 1983), 74-80, n" 8.

19 L'inventaire est cité par A. Darcel ("Le Moyen Age ", 1007) qui l'appliqua par erreur à la tapisserie des Puttini qui nous occupe. Cette assertion est à l'origine de la croyance erronnée que le Vatican possède aussi une telle tenture des Puttini (cf. Forti, Ferrara, 76, corrigé par Adelson, Burlington Magazine [May 1985], 308). Reproduction chez Erkelens, "Grotesken ", 125, et F. Viatte, Le XVI" siècle européen. Tapisseries, cat. expos. (Paris, 1965-1966), 38 et 51, nº 28; pour la tenture de Geubels, cf. E. Duverger, "Tapijtwerk uit het atelier van Frans Geubels ", dans L'Age d'or de la tapisserie flamande (Bruxelles, 1969), 184-92; pour celle de Coypel: M. Fenaille, Etat général de la Manufacture des Gobelins (Paris, 1903), II, 224.

20 Ainsi, sur une pièce autrefois au Kunstgewerbemuseum de Berlin, H. Schmitz, Bildteppiche (Berlin, 1919), fig. 156; Boccardo, "Raffaello ", fig. 77), et sur la tenture bruxelloise des Dieux Grotesques, vers 1545, à Vienne (E. Scheicher, "Die Groteskenmonate ", Jahrbuch der Kunsthistorischen Sammlungen in Wien, LxIx [1973], fig. xxx).

21 Ainsi, Saint Augustin recommande d'appeler l'aide divine "contra illos fluctus procellasque fortunae" (cité par K. Heitmann, Fortuna und Virtus. Eine Studie zu Petrarcas Lebensweisheit [Cologne, 1958], 20). Boèce, qui créa l'archétype de la Fortune médiévale tournant la roue, avertit souvent contre les dangers marins ( $\mathrm{e}$ Consolatione Philosophiae II, I, pr. 55; II, III, v. 9-12; II, IV, v. 5-6) ce qui suscitera de nombreuses descriptions de la Fortune marine, entre autres celles du château de Fortune en mer, par ex. chez Alain de Lille, ensuite dans le Roman de la Rose (vv. 5920-6118) et chez Pétrarque (cf. Heitmann, Fortuna und Virtus, 27, 69-70)

22 De Tervarent, Attributs, 283, cite le pavement de la cathédrale de Sienne selon Pinturicchio, un dessin de Van Orley, une gravure de H.S. Beham. Pour la "Fortuna iconographie doit beaucoup à la philosophie de l'époque. Chez d'innombrables auteurs, depuis Alberti et Valla jusqu'à Ficino, Pico della Mirandola et surtout Pontano et son De Fortuna Libri 3 , paru chez Aldo Manucci en 1518, la Fortune n'est plus seulement crainte comme une puissance instable et nocive, mais l'homme est invité de prendre son sort en main, de tenir le gouvernail de sa vie, de nager à contre-courant et de se ranger sous la bonne Fortune ${ }^{23}$.

Tout ceci nous incite à proposer une polysémie pour cette remarquable tapisserie. Vénus n'estelle ici pas aussi la bonne Fortune, tenant le gouvernail de la barque de l'Etat, et voguant sous l'égide du cardinal? Ce dernier est présent non seulement par ses armoiries dans la bordure supérieure, mais aussi et surtout par le génie inscrivant son nom (HERcules EPISCOP(?) MANtovanus) sur la tablette à l'avant-plan, une image associant le commanditaire non seulement à la Fortune politique mais aussi à la Renommée (Fig. $84)^{24}$. La tenture des Puttini ne nous étant parvenue que partiellement, il n'est pas possible d'affirmer si ce thème se poursuivait sur les autres tapisseries. Peut-être est-il sous-jacent dans le Jeu de Balle (Fig. 80), motif présent chez Philostrate mais pouvant être associé à l'attribut versatile de la Fortune.

De nombreux détails de cette tenture diffèrent par ailleurs des thèmes habituels de la Fortune et des descriptions classiques de Philostrate. Ils se prêtent à d'autres polysémies, aussi bien orientées vers la tradition païenne que vers un sens chrétien et " liturgique " plus ou moins explicite. En effet, à l'opposé des Images où les érotes récoltent seulement des pommes, les petits amours de cette tenture cueillent surtout des grappes de raisins, qui poussent dans des vignes " sauvages ", grimpant le

velata " de la Renaissance, voir F. P. Pickering, Literalure E Art in the Middle Ages (Londres, 1970), 219-20, et Ph. Verdier, "Sur trois oeuvres de Léonard Limousin ", Revue de l'Art, LXxvir (1987), 52-54.

23 Cf. A. Doren, "Fortuna im Mittelalter und in der Renaissance ", Vorträge der Bibliothek Warburg. 1922-1923, I (1924) 120-36; E. Cassirer, Individuum und Kosmos in der Philosophie der Renaissance (Leipzig et Berlin, 1927), 81-92; A. Warburg, "Francesco Sassettis letztwillige Verfügung ", $G e$ sammelte Schriften (Leipzig et Berlin, 1932), I, 149-50 et fig 36.

24 Le deuxième mot abrégé sur la tablette, entre HER(cules) et MAN(tovanus) est fortement restauré, mais pourrait être EPISCO(pus). La figure bien connue de la Victoire écrivant le nom du victorieux sur un bouclier fut empruntée à la Colonne Trajane; on la rencontre par exemple dans le Codex Escurialensis et sur les bords de la tenture des Fructus Belli, tissée en 1546-1548 pour Ferrante Gonzaga, frère d'Ercole (G. Delmarcel, "Fructus Belli, une tenture bruxelloise pour Ferrante Gonzaga ", Bulletin de Liaison du Centre international d'Etude des textiles anciens, n* 59-60 [1984], 4253) 
long des troncs d'arbres de lauriers et de châtaigniers. Ce thème des génies ailés dans la vigne, motif dionysiaque remontant entre autres à des reliefs de sarcophages antiques ${ }^{25}$, était familier en Italie du Nord dès le Quattrocento. Signalons ainsi une estampe, attribuée au Maître $\mathbf{E}$ du Tarot, un artiste actif à Ferrare vers 1465, et illustrant une telle composition ${ }^{26}$. Une même image apparaît dans Le Songe de Poliphile de Fra Francesco Colonna (édition Venise 1499): on y voit les putti récoltant les raisins et pressant le vin, ainsi que le putto urinant. Dans le symbolisme funéraire des Romains, le vin était un breuvage d'immortalité27. Le duc Federico aussi bien que le cardinal Ercole peuvent s'être plus à commander une telle représentation en tapisserie, mais pour ce dernier, elle pouvait également se prêter à une allusion eucharistique, la vigne se référant au vin du sacrifice de la Messe, bien appropriée pour un prince de l'église. A ceci s'ajoute que les amours cueillent aussi des grenades, anciens symboles chrétiens de bonheur, de victoire et de vie éternelle ${ }^{28}$.

Enfin et surtout, la morphologie de la Barque de Vénus/Fortune (Fig. 79) peut offrir une clef de lecture bien intéressante. La Fortune dirige en effet une barque dans laquelle pas moins de dix petits génies s'occupent de la pêche au filet. La barque vogue sous une pergola sur laquelle pousse une vigne et où d'autres génies chassent des oiseaux. Hormis le génie inscrivant le nom d'Ercole sur la tablette, l'avant-plan est surtout occupé par des oiseaux aquatiques. Les traits principaux de cette composition nous semblent tellement apparentés à ceux de la tapisserie de la Pêche Miraculeuse, dans la tenture des Actes des Apôtres conçue par Raphaël en 1514-1516 sur ordre du pape Léon $x$, que nous sommes enclins d'y voir plus qu'une simple coïncidence : la Vénus/Fortune prend la place du Christ, le génie qui l'embrasse évoque Pierre s'agenouillant, les génies pêcheurs et même les hérons et les grues de la rive renvoyent directement cette fameuse tapisserie, dont le cardinal Ercole acquit d'ailleurs plus tard une édition (comparez Fig. 79 et Fig. 86). Ne peut-on dès lors considérer les génies pêcheurs comme des re-

25 F. Matz, Die dionysischen Sarkophage (Berlin, 1969), iI, 327, $n^{\circ} 178$ : seul sarcophage montrant une vendange où des amours assistent au travail, et où ils pressent le vin, sur le couvercle.

$26 \mathrm{Ch}$. Mesenzeva, "Zum Problem: Dürer und die Antike. Albrecht Dürers Kupferstich "Die Hexe" ", Zeitschrift für Kunstgeschichte, xLvI (1983), 193 et fig. 7, p. 192.

27 G. de Schoutheete de Tervarent, "L'origine des fontaines anthropomorphes ", Académie royale de Belgique. Bulletin de la Classe des Beaux-Arts, xxxvin (1956), 127-28 et fig. 16.

28 Pour la grenade comme symbole de bonheur et de victoire dans l'art paléochrétien, cf. F. Muthmann, Der Granatapfel. Symbol des Lebens in der alten Welt (Berne, 1982), 113 et ss. présentants des apôtres, pêcheurs d'âmes, voguant sous la tonnelle du vignoble eucharistique? Dans un même sens peuvent alors s'interpréter les génies dans les vignes des tapisseries suivantes, aussi ceux occupés de la pêche, même ceux dansant sous la vigne et dont l'un est touché par une flèche tombant du carquois (la grâce frappant l'âme du païen ingénu?).

Il nous semble en effet légitime de supposer que le cardinal Ercole ait voulu conférer un sens bien précis à cette tenture des Jeux d'Enfants, sinon dès le départ - si la tenture était conçue initialement pour son frère-du moins lors de l'élaboration de la plus grande part des dix tapisseries figurées. Le parangon du genre était en effet la tenture des vingt Putti aux Armes de Léon $x$, conçue peut-être par Giovanni da Udine mais dont les cartons furent dessinés et peints sur place à Bruxelles par Tommaso Vincidor en 1521 pour y être tissés ensuite par Pierre van Aelst, le fabricant des Actes. Les recherches récentes ont prouvé clairement que ces "bigarie [=bizarrie] de putini, cose alegre acomodate [sic] per tute le vostre imprese ", tel que Vincidor l'écrit au Pape, ont été conçues selon un programme servant à glorifier les faits et qualités de Léon $\mathrm{x}$ Médicis par des attributs surtout héraldiques, et selon un format strictement adapté au cérémonial auquel elles étaient destinées, à savoir la décoration de la zône inférieure de la Salle de Constantin, la salle d'apparat pour de grands banquets officiels (Fig. 87) ${ }^{29}$.

Le remploi profane de la Pêche Miraculeuse de Raphaël ne doit pas nous étonner sur cette tenture conçue par Giulio vers 1540. N'oublions pas que l'artiste a commencé sa carrière dans l'atelier de Raphaël précisément à l'époque de la réalisation des cartons des Actes des Apôtres, probablement de la fin de 1514 à la fin de 1516 . Hartt est même enclin d'attribuer à Giulio la plus grande part de l'agrandissement des modèles en dessin à la craie noire, préparant ainsi la composition peinte ensuite par Raphaël lui-même. Et c'est aussi dans cette Salle de Constantin que Giulio travailla après la mort de Raphaël, de 1520 jusqu'à l'achèvement en 1524, quittant Rome ensuite pour Mantoue ${ }^{30}$.

29 N. Dacos, "Tommaso Vincidor, un élève de Raphaël aux Pays-Bas ", dans Relations artistiques entre les Pays-Bas et l'Italie à la Renaissance. Etudes dédiées à Suzanne Sulzberger (Bruxelles et Rome, 1980), 63 et 95. Pour les Jeux d'Enfants de Leon x voir R. Quednau, "Zeremonie und Festdekor. Ein Beispiel aus dem Pontifikat Leos X. ", dans Europäische Hofkultur im 16. und 17. Jahrhunderts, Kongress 1979 (Hambourg, 1982), II, 349-58, et R. Quednau dans Raffaello in Vaticano, cat. expos. Città del Vaticano, 1984-85 (Milan, 1984), 357-63, $\mathrm{n}^{\circ} 135$. Cette tenture a influencé des tapisseries flamandes. Cf. G. Delmarcel, Tapisseries d'Enghien du $X V I^{\mathbb{E}}$ siècle. Verdures avec Jeux d'Enfants, Musées vivants de Wallonie et de Bruxelles, 6 (Liège, 1983).

30 Hartt, Romano, I, 18 et 42-51. 
Le schéma de composition adopté ici par Giulio pour les Puttini du cardinal Ercole remonte à des sources diverses que l'artiste doit avoir connues. Hormis les exemples plus anciens des estampes mentionnées plus haut, il faut surtout se rappeler des dessins et des xylographies au monogramme NDB, issus de la "bottegha " de Raphaël et reflétant une composition du maître, où l'on voit les génies cueillant les pommes dans une treille, selon le texte de Philostrate et dans une mise en page qui offre la source directe des tapisseries décrites ici ${ }^{31}$. Des exemples de tels génies sont signalés dans des oeuvres de Giulio avant 1539. On sait que des assistants de Giulio sont occupés à peindre des puttini avec des feuilles de vigne sur fond bleu, dans une pièce située près du " giardino secreto" du Palazzo del Te en janvier de 1532. Un dessin conservé à Chatsworth en serait un reflet ${ }^{32}$.

En créant cette tenture des Jeux d'Enfants vers 1540 , d'abord peut-être pour le duc Federico, ensuite et sûrement pour le cardinal Ercole Gonzaga, Giulio s'est donc souvenu des ensembles majeurs de tapisseries auxquels il fut confronté durant ses années romaines: les Actes des Apôtres, les Grotesques avec Divinités et les Jeux d'Enfants de Léon $\mathrm{x}$, toutes conçues dans l'atelier de Raphaël dont il fit partie ${ }^{33}$. Tout comme Vincidor l'avait fait pour le pape, Giulio sut allier le thème antique des Erotes de Philostrate, suggérant une vie de plaisir et de luxe dans une ambiance païenne, aux exigences d'un prince de l'Eglise, dont il évoque ici-au moins sur une des quatre pièces conservées - la bonne Fortune quittant le rivage, avec la mission de pêcher des hommes pour le Christ et de se souvenir de lui par le sacrifice eucharistique.

31 Reprod. dans Raffaello in Vaticano, 357-58. Quednau les cite à propos des Jeux d'Enfants de Léon $\mathbf{x}$, mais ces oeuvres sont beaucoup plus apparentées aux Puttini d'Ercole! Voir aussi Raphaël dans les collections françaises, cat. expos. (Paris, 1983-1984), 392, $\mathrm{n}^{\circ} 90$.

32 Hartt, Romano, I, 159 (palais du Té), et II, fig. 354, cat. 217 (dessin Chatsworth)

33 On ignore la date exacte de l'achèvement du tissage des Puttini de Léon $x$. Par le contrat établi le 27 juin 1520 , Pieter van Aelst reçut la promesse d'un salaire de 3600 ducats, à raison de 100 ducats par mois, ce qui suppose trois années de travail (W. Bombe, "Raffaels Teppiche und Pieter van Aelst ", Repertorium für Kunstwissenschaft, L [1920], 18-19). Les cartons étant dessinés par Vincidor en 1521, on peut supposer que la tenture était à Rome avant le départ de Giulio pour Mantoue en octobre 1524. Notons en tous cas que la plus ancienne réédition connue de cette tenture est apparemment la pièce - isolée, ou fragment d'une série perdue?-issue du second atelier de Nicolas Karcher à Mantoue (1555-1562), et pourvue des armoiries de Guglielmo Gonzaga, duc de Mantoue de 1550 à 1587 . C'est un témoignage, quoique tardif, de l'intérêt direct des Gonzague pour cette tenture (cf. Forti, Ferrara, 79 et 189).

\section{LES PUTTINI DE FERRANTE GONZAGA, SEIGNEUR DE GUASTALLA}

La tenture des Jeux d'Enfants, créée par Giulio Romano pour le cardinal Ercole Gonzaga et exécutée selon toute probabilité à Mantoue même par le licier bruxellois Nicolas Karcher entre 1539 et 1545 , semble avoir généré une tenture similaire, mais non identique, qui fut tissée à Bruxelles entre 1552 et 1557 pour son frère puîné, Ferrante Gonzaga (1507-1557), duc d'Ariano, généralissime des armées impériales, gouverneur de Milan de 1546 à 1554 et fondateur (1539) de la branche cadette des Gonzaga di Guastalla ${ }^{34}$. L'histoire de cette tenture, conservée actuellement dans la collection du comte Giannino Marzotto (Figs 88-94), n'a jamais été établi clairement, non plus que les relations formelles et iconographiques avec la tenture précitée.

Le premier inventaire général des tapisseries des Gonzaga di Guastalla, datant de 1590 et établi sous Ferrante II, petit-fils de Ferrante I, mentionne sous le numéro 13: "Tapezzaria delle Puttini con oro et argento, alta ale cinque, pezzi sei ". D'après ce document, la tenture des Puttini est la seule des vingt-deux séries cataloguées qui contienne de l'or et argent. Le détail n'est pas sans importance $^{35}$.

C'est grâce à une correspondance menée par le cardinal de Granvelle en 1571-1574 que nous savons que ces six Jeux d'Enfants d'or et argent ont été commandés par Ferrante I Gonzaga. Antoine Perrenot de Granvelle, premier archévêque de Malines, se constitua une importante collection de tapisseries durant son bref séjour dans les PaysBas de 1561 à 1564 . Il continua de s'intéresser aux tapisseries flamandes durant son exil à Madrid, et il plaça de nouvelles commandes à Bruxelles à partir de 1571, quand il fut nommé vice-roi de Naples. Les lettres qui lui furent envoyées par ses secrétaires et agents Viron et Morillon nous permettent de suivre l'élaboration de quelques-unes de ces tentures ${ }^{36}$. La plupart sinon toutes furent commandées auprès de l'atelier de Willem de Pannemaker, le marchand-tapissier le plus célèbre de Bruxelles, et le fournisseur régulier tant de la cour de Charles-Quint que de celle de Philippe II.

Dès la fin de 1570 , le cardinal doit avoir demandé de retrouver les patrons d'une " tapisserie

34 A propos de ses tapisseries, voir Delmarcel, "Fructus Belli ", 42-53.

35 L'inventaire de Guastalla (1590) sera publié ultérieurement (cf. note 1).

36 Nous citons les extraits de la correspondance de Granvelle concernant les tapisseries des Gonzague (Mss Granvelle de la bibliothèque de Besançon) d'après Maurice Piquard, "Le cardinal de Granvelle, amateur de tapisserie ", Revue belge d'archéologie et d'histoire de l'art, xix (1950), 121-25. 
des Vignes ». Le 16 février 1571, Viron fait savoir "que Pannemaker n'en avait aucun patron ", mais le 24 prochain, il précise: "Et quant a la tapisserie du Vignoble de don Fernand de Gonzaga, ledict Pannemaker a tant sercher [sic] par ses papiers qu'il a trouvé une piece du bordz d'icelle que va icy aussy jointement, et du surplus il le fauldroit sercher a ceulx qui l'ont emportez avec ladicte tapisserie ". On peut en déduire que Granvelle se souvenait d'une tapisserie des Vignes qui avait appartenu à Ferrante Gonzaga, que Pannemaker en a retrouvé le dessin de la bordure, et que les patrons des pièces mêmes ont été emportés par d'autres. Viron (et Pannemaker?) continuent leurs recherches et environ un an plus tard, le 29 juin 1572, Viron signale à son maître : "De celle [tapisserie] de la Vigne et Petiz Enfant, j'ay avertir v.s.I. ou sont les patrons dont je tiens se pourront recouvrer par v.s. des Goncages ". Les Gonzague possèdent donc les patrons ou cartons de grandeur nature.

Granvelle abandonne ensuite le projet pour un certain temps, mais l'idée est reprise au début de 1573 , quand Viron répète à son maître : « Et quant à l'aultre tapisserie de don Fernando de Gonzaga je suis este devers ledict Pannemaker qui dit n'a les patrons d'icelle et que les heritiers dudict don Fernando les ont emporté ". A l'instigation de Granvelle, Viron doit avoir écrit à Cesare Gonzaga, fils de Ferrante et son héritier, et le $\mathrm{I}^{\mathrm{er}}$ octobre, il peut signaler au cardinal: "j'ay receu la lettre du $\mathrm{Sr}$ Cesar de Gonzagua en vertu de laquelle le marchant nommé Nicole m'a accorde me delivrer les patrons, combien qu'il dit l'on luy est redevable de IV ou v cent escuz ". Viron avertira Granvelle du nombre de patrons, et il suggère que l'on y ajoute des " oiseaux au vifz, faiz par More " ainsi que les armes de Granvelle.

Dès leur remise à Willem de Pannemaker, les cartons doivent avoir été mis sur le métier et les tapisseries furent tissées sans plus tarder : le 9 janvier 1574 , Viron précise qu'il y aura six pièces, de cinq aunes et demi de hauteur $(=380 \mathrm{cms})$; le 31 mai, il confirme que le travail est en cours, et le 4 juillet, il signale que la tapisserie de la Vigne va être livrée et envoyée, sauf la dernière pièce.

Il n'est pas étonnant que Granvelle se soit souvenu en 1570 d'une tenture faite pour Ferrante, donc avant la mort de ce dernier en 1557. Les rapports entre l'archévêque de Malines et le généralissime des troupes impériales furent fréquents, même s'ils n'étaient pas toujours des plus amicaux ${ }^{37}$. Une tradition bien établie, et pro-

37 Ferrante fut nommé chef d'état-major de l'armée impériale sur proposition du père de Granvelle, le chancelier Nicolas Perrenot, en 1543; la paix de Crépy (1544) fut surtout l'oeuvre de Granvelle et de Ferrante, mais leurs relations se bablement exacte, veut que ces six tapisseries de la Vigne et petiz Enfant furent achetés par le roi Philippe après le décès du cardinal, en 1586 , et que quatre pièces existent encore de nos jours dans les collections royales d'Espagne ${ }^{38}$. Pourvues de fils d'or et d'argent et de la marque de Willem de Pannemaker, elles présentent des bambins nus jouant dans une vigne et s'occupant surtout d'y cueillir des raisins. La bordure-cette bordure dont de Pannemaker possédait encore un fragment en 1571 - est ornée d'une frise de pampres et de grappes, interrompue à endroits réguliers par des têtes de faunes et de satyres.

Ces tapisseries, qui d'après les lettres citées furent copiées de cartons plus anciens d'une tenture ayant été commandé initialement par Ferrante Gonzaga, ressemblent en tous points à celles formant encore une série complète de six pièces, munies de bordures et de marques identiques et présentant les mêmes compositions (Figs 88 à 94). Nous sommes ici, sans aucun doute, en présence de la tenture originale de Ferrante, qui resta dans la possession des Gonzague jusqu'au xvirI ${ }^{\mathrm{e}}$ siècle, quand elle passa aux mains d'autres familles nobles d'Italie du Nord. Elle y resta jusqu'au $\mathbf{x x}^{\mathrm{e}}$ siècle, entre autres chez les Dona dalle Rose en $1935^{39}$.

D'autres documents, italiens cette fois, viennent confirmer et préciser l'histoire de cette splendide série de tapisseries. Une lettre du peintre Gian Battista Lodi da Cremona, envoyée de sa résidence de Lierre près d'Anvers le 5 février 1552 à Ferrante Gonzaga, se rapporte sans aucun doute à la commande de ces six Puttini ${ }^{40}$. Lodi, qui agissait

détériorèrent après la disgrâce de Ferrante à Milan. Rappelons que Leone Leoni fut introduit à la cour de Bruxelles sur recommandation commune de Granvelle et de Ferrante; cf. M. Van Durme, Antoon Perrenot, bisschop van Atrecht, kardinaal van Granvelle, minister van Karel $v$ en van Filips II (1517-1586) (Bruxelles, 1953), 30, 34, 108, 113, 240 et passim.

38 P. Junquera de Vega et C. Herrero Carretero, Catalogo de Tapices del Patrimonio Nacional, vol. I: Siglo xvl (Madrid, 1986), 236-40 (serie 33 : juegos de Niños). L'inventaire des tapisseries établi après la mort de Philippe II, en 1598, cite cette tenture "de unas parras con unas figuras de unos niños desnudos "(en 7 pièces, un sujet étant dédoublé) sans autres précisions, mais juste après une tenture en 12 pièces " de unas galerias de jardines [...] que son los que por el mes de septiembre del ano de 1587 se compraron de la almoneda del Cardenal Granvela " (Madrid, Archivo del Palacio Real, leg. 919, fol. 107).

39 M. Crick-Kuntziger, "Tapisseries ", Cinq siècles d'art. Exposition universelle de Bruxelles (Bruxelles, 1935), 83, $\mathrm{n}^{\circ} 648$. La tenture fut vendue peu après à la famille Marzotto. Les six tapisseries, en excellent état de conservation, sont tissées en fils d'or, d'argent, de soie et de laine; finesse du tissage: huit à neuf fils de chaîne au centimètre; elles ont une hauteur de 383 à $390 \mathrm{~cm}$ et une largeur de 415 à $473 \mathrm{~cm}$.

40 Voir notre Document I, déjà publié par J. A. F. Orbaan, "Parmezaansche Gegevens ", Oud Holland, xLI (19231924), 252-53, et cité ensuite par B. Meijer, "Cremona e i 
aussi à d'autres occasions comme l'agent artistique de Ferrante à Bruxelles ${ }^{4}$, signale au gouverneur de Milan qu'il est allé à Bruxelles, selon ses instructions, pour les tapisseries "da farse subito ", et qu'il y a trouvé un tapissier ayant de nombreux et de bons ouvriers, "il quale per la Cesarea Maestà riduce in tapezzaria d'oro l'impresa di Tunis ». Il s'agit donc de Willem de Pannemaker, qui tissa effectivement dans ses ateliers, de 1548 au 12 avril 1554 , la fameuse tenture de la Bataille de Tunis d'après des maquettes et des cartons de Jean Vermeyen $^{42}$. Lodi ne cite pas le sujet des tapisseries commandées, mais celles-ci doivent contenir des fils d'or et d'argent. Le prix demandé était de quatorze florins par aune, mais seulement de huit si le commanditaire livre l'or lui-même, et Lodi déconseille de mettre des fils d'argent, qui s'oxydent par la suite. A propos de Pannemaker, Lodi note en passant : " egli ha fama del miglior mastro che ivi se trove ", un jugement encore valable de nos jours.

En confrontant cette lettre aux documents cités plus haut, on peut conclure qu'il s'agit ici de la commande des Puttini, car: l) la seule tenture à fils d'or et argent en possession des Gonzaga di Guastalla en 1590 est celle des Puttini; 2) Granvelle fait copier par Pannemaker en 1570-1574 une tenture de la Vigne en six pièces qui appartint à Ferrante Gonzaga, et 3 ) les restes de cette réédition pour Granvelle, à présent à Madrid, sont identiques aux six pièces de la collection Marzotto, munies de la même bordure et du monogramme de Pannemaker. La tenture des six Puttini de la collection Marzotto a donc été tissée pour Ferrante Gonzaga entre février 1552 et le 15 novembre 1557, date de son décès à Bruxelles.

Les Gonzaga di Guastalla ont toujours pris un soin extrême de cette splendide tenture qui leur était parvenue de leur aïeul. Les documents relatant leurs vicissitudes jusqu'au début du xvrrI ${ }^{\mathrm{e}}$ siècle en témoignent. Résumons ici les textes les plus intéressants. Cesare Gonzaga, le fils de Ferrante I, connut durant toute sa vie de graves difficultés financières qui l'obligèrent à hypothéquer constamment les oeuvres d'art qu'il possédait. Ainsi, il fut forcé à maintes reprises de donner des tapisseries en gage, une pratique qui était courante

Paesi Bassi ", I Campi e la cultura artistica cremonese del Cinquecento (cat. expos., Crémone, 1985), 25-26. Ces auteurs n'établissent toutefois pas la relation avec la tenture des Puttini.

41 C'est probablement lui le "Jehan Baptiste painctre " cité par le tapissier Jean Baudouyn, le 31 août 1547, à propos de la tenture des Fructus Belli qu'il tissait pour Ferrante (cf. Delmarcel, "Fructus Belli ", 43 et note 12).

42 Junquera et Herrero, Catalogo, 73, série 13. La parution de la monographie de J. H. Horn (de l'Université de Guelph) sur J. C. Vermeyen et la tenture de Tunis est imminente. dans les familles nobles car les tentures représentaient un capital mobile considérable. Cesare suivit ainsi l'exemple de son père Ferrante, qui dut engager une partie de sa collection de tapisseries à une date non précisée, afin de pouvoir disposer de quatre à cinq mille ducats pour fortifier les murs de la ville de Milan (ASPr, Busta 50/1). Quant à Cesare, on sait qu'il engagea des tapisseries en 1561 et 1562, mais la tenture des Puttini resta entre ses mains, car en octobre 1561 il en refuse le prêt temporaire au cardinal Simonetta (ASPr, Busta 46). En septembre de 1567, il dut donner aussi les Puttini en gage aux banquiers vénétiens Paolo Moro et Bartolomeo Calice, en même temps que d'autres tapisseries précieuses de la famille, telles que les Fructus Belli et que sa collection d'argenterie et de gemmes (вма, Busta 23, сс. 36-55). Cette situation pécuniaire difficile explique aussi que Cesare fut disposé à vendre les cartons originaux des Puttini, encore en sa possesion, au cardinal Granvelle en 1573, comme nous l'avons constaté plus haut.

Cesare, décédé le 17 février 1575 , ne réussit pas à récupérer de son vivant ses tentures engagées, car son fils Ferrante in dut encore les demander en prêt, tant les Puttini que les Fructus Belli, pour son propre mariage avec Vittoria Doria en 1587 (ASPr, Busta 50/2, cc. 55). Dans cette même période se situe un épisode bien curieux pour l'histoire des deux tentures des Jeux d'Enfants des Gonzague. Nous avons signalé que le cardinal Ercole légua en 1563 sa tenture italienne des Puttini (Figs 79-82) à son neveu Guglielmo Gonzaga, le duc de Mantoue. Un document issu par Guglielmo, non daté mais rédigé avant son décès en 1587 , contient des instructions à un certain "Modone hebreo ", enjoignant ce dernier à se rendre à Venise pour y aller examiner "la tapezzaria che ha colla Sua Eccellenza Don Ferrante Gonzaga, chiamato delli Puttini " ${ }^{43}$. Il doit la comparer à la tenture du même nom en possession du duc. Modone devait même emporter une pièce de la tenture de Guglielmo, pour voir si celle de Ferrante pourrait compléter l'autre. Au cas échéant, il doit essayer d'en acheter une pièce auprès des détenteurs de cette série, donnée en gage par Cesare en 1567. On peut en déduire que l'une ou l'autre pièce de la tenture italienne du cardinal Ercole, passée ensuite au duc de Mantoue, s'était perdue ou endommagée, et que Guglielmo se proposait de la compléter par une tapisserie de la tenture bruxelloise de son autre oncle. Cet achat ne s'accomplit toutefois pas, car l'inventaire général précité des tapisseries présentes à Guastalla en 1590 mentionne la série complète des six pièces, qui furent

43 Voyez notre Document II. 
donc récupérées des mains des créanciers par Ferrante II après son mariage en 1587.

La tenture bruxelloise des Jeux d'Enfants resta par la suite chez les Gonzaga di Guastalla jusqu'en 1670. En cette année, Ferrante in donna sa fille Anna Isabella en mariage à son parent Ferdinando Carlo, duc de Mantoue. Il la pourvut d'une dot considérable de 200000 écus, constituée en partie par des joyaux, de l'argenterie et des tapisseries : " e questi in tante supellettili dalla più pretiose come sono li apparati nominati Fructus Belli e li Putini $" 4$. Ainsi, les deux tentures les plus précieuses des Gonzaga di Guastalla passèrent à la branche majeure de la famille. Après le décès du dernier duc de Guastalla, Ferrante III, en 1678, le patrimoine artistique de cette famille fut divisé en parties égales entre les héritiers. Anna Isabella y reçut la confirmation de ses droits sur les oeuvres d'art qui lui furent données en dot. Ainsi, la tenture des Puttini fut estimée à 22500 écus, seulement dépassée par les tentures de l'Histoire de Moïse, douze pièces évaluées à 45000 écus, et les Fructus Belli, huit énormes pièces taxées à 75000 écus bien qu'elles ne contiennent pas de métal précieux ${ }^{45}$.

Quand Ferdinando Carlo, le mari d'Anna Isabella, décéda le 5 juillet 1708 à Padoue, la branche majeure des Gonzague ducs de Mantoue s'éteignit à son tour. L'inventaire de ses biens établi par la seigneurie de Venise en janvier de 1709, contient la dernière mention des deux tentures des Jeux d'Enfants en possession des Gonzague que nous étudions ici : d'une part les dix pièces "tessuti con oro, disegno di Giulio Romano ", celles du cardinal Ercole, de l'autre "pezzi di arazzi grandi numero 6 instoriatti con Puttini tessuti con oro, disegno di Raffael " ou celles de Ferrante I de Guastalla $^{46}$. Tous les biens de Ferdinando Carlo, les tapisseries inclues, furent ensuite remis par la Quarantia Criminale de Venise à la famille de son épouse, donc au duc de Lorraine ${ }^{47}$. Ce dernier les a apparemment revendues, car la tenture des Jeux d'Enfants se trouve par la suite aux mains de plusieurs familles nobles apparentées en Italie du Nord: les Barbarigo, les Michiel, Martinengo et Donà dalle Rose. Elle est citée dans un inventaire, daté du 10 octobre 1762 du Palazzo Sagredo à Venise, estimée alors à 590 zecchini, et mentionnée dans un inventaire ultérieur comme "Pezzi sei di

44 ASMn, Busta 214, cc. 258-61; Braghirolli, Arazzi in Mantova, 49-50.

45 asmn, Busta 214, fol. 367; Braghirolli, Arazzi in Mantova, $51-52$.

46 ASmn, Busta 400 (copic de l'original aux archives de Vérone).

47 A. Luzio, La Galleria dei Gonzaga (Milan, 1913), 85-86 et 316-31. arazzi di Raffael d'Urbino, tessuti d'oro con Baccanali di Puttini che distaca uva e scherza tra loro indica l'Autunno $"$ 48.

Ces dernières mentions de la tenture sous l'Ancien Régime nous amènent aux problèmes d'iconographie et d'attribution artistique de l'ensemble. Chacune des six tapisseries de cette tenture présente la même ordonnance. Le champ visuel est divisé en trois parties par des chênes disposés deux à deux et reliés entre eux par des ceps s'enroulant autour des troncs d'arbres et de leurs branches, chargés de grappes mûres de raisins. Ces vignes en forme de tonnelle ou de pergola sont envahies par des bambins nus cueillant les grappes et s'adonnant à quelques activités, qui permettent d'intituler chaque tapisserie.

La tapisserie de la Ville (Fig. 89) se distingue par le décor architectural de l'arrière-plan, montrant des demeures seigneuriales et un campanile le long d'une rue, ceci à l'opposé des cinq autres pièces, où le fond aboutit sur une perspective plutôt rurale. Les trois bambins du groupe central semblent jouer à saute-mouton, ou montent à cheval sur le dos de l'un d'eux tandis que leurs compagnons se transmettent les grappes cueillies. La tapisserie du Lièvre (Fig. 90) mérite ce nom par la dispute de quatre génies autour d'un grand lièvre, tandis que leurs compagnons cueillent et transportent le raisin. Sur le Cheval-Bâton (Fig. 91 ), un des enfants manie ce jouet, aux côtes de ses compagnons qui tiennent une coupe de raisins; les bambins à gauche semblent discuter à propos d'un vase tenu par l'un d'eux; à droite, un génie tient une hampe cruciforme où flotte une bannière. Sur la quatrième pièce où l'on assiste à la récolte du raisin, les enfants du groupe central mènent un Bouc (Fig. 92) par la laisse. Les petits vignerons, portant leurs grappes dans des paniers ou dans des riches plats d'orfèvrerie, apportent finalement leur récolte au Pressoir (Fig. 93), où les raisins sont pressés. Enfin, tandis que quelques génies cueillent encore des grappes dans les frondaisons, leurs compagnons mènent la Danse (Fig. 94) sous la tonnelle, pour célèbrer la fin des vendanges. Trois enfants s'amusent à lutter à gauche, tandis qu'un petit compagnon blessé ou fatigué est transporté sur la droite.

En décrivant les six pièces, nous avons proposé en même temps une séquence de lecture, toute

48 G. Lorenzetti et L. Planiscig, La collezione dei conti Donà dalle Rose a Venezia (Venise, 1934), 69-70), n"341-44. Voir en outre Venise, Biblioteca Correr, Ms. PD.C.2753, viı, 1755 : "Inventario del Eccellentissima Casa Sagreda (16 settembre) ", et Ms. PD.C. 2755, II, 1763, "Nota degli arazzi appresi in pagamento di dote (30 mar7o) ". En 1934, seules quatre des six pièces étaient conservées ensemble; les deux autres les ont rejoint par la suite, et la tenture est de nouveau complète de nos jours. 
provisoire soit-elle. Si ces tapisseries furent destinées à être pendues-et donc lues-dans un certain ordre, elles peuvent avoir été ordonnées selon le déroulement de l'action: les quatre premières offrent des scènes de vendanges, qui mènent à la confection du vin dans le Pressoir. La vendange et le pressage terminés, les génies vendangeurs s'adonnent à la Danse. Ce progrès de l'action trouve peut-être un écho formel dans le nombre croissant de chênes constituant la pergola des vignes : respectivement quatre arbres dans les trois premières, six dans le Bouc, huit dans le Pressoir, enfin toute une allée dans la Danse.

On constate d'emblée que, comme dans la tenture italienne du cardinal Ercole, ces Jeux d'Enfants tissés à Bruxelles ne contiennent que quelques allusions directes au chapitre de Philostrate: seules la présence d'enfants nus dans un verger, leur chasse du lièvre, la lutte et la danse s'y rallient directement. Les changements et variations y sont tout aussi nombreux. Les génies ne portent pas d'ailes et ils ne cueillent nulle part des pommes. Ils ne sont pas des serviteurs de Vénus, mais de Bacchus : la cueillette des raisins mène à la vendange, et le bouc est une de ses incarnations ${ }^{49}$. Cette vendange semble quelque peu christianisée sur le Cheval Bâton (Fig. 91) par la bannière christologique, portée normalement par St. Jean Baptiste, et par le vase pouvant faire allusion au calice. Remarquons aussi que cette tenture n'a presque pas de liens thématiques directs avec les Jeux d'Enfants du cardinal Ercole. Les deux ensembles puisent à une même veine générale d'inspiration, conceptuelle et formelle, mais leur développement est tout différent. On ne peut dès lors pas prétendre que les Puttini bruxellois de Ferrante soient une copie de la tenture italienne d'Ercole ${ }^{50}$.

Mieux vaut peut-être de considérer le thème général de cette tenture. De cette vigne bucolique habitée par tant de bambins innocents et insouciants se dégage avant tout un sens paradisiaque. La joie de vivre dans la nature est accouplée à celle procurée par le vin et à la jeunesse. La combinaison de la vigne et des frondaisons des chênes, dans des paysages campagnards où les villes se profilent parfois à l'arrière-plan, peut évoquer ici le locus amoenus si cher à la Renaissance, la vallée de Tempe selon les descriptions d'Elien et de Pline ${ }^{51}$.

49 Le jeu cultique des amours et du bouc dionysiaque est déjà représenté sur une gravure italienne, peut-être ferraraise, de la fin $d u x^{e}$ siècle; $c f$. Mesenzeva, "Dürer [...] die Hexe ", 192-93.

50 Forti, Arazzi a Ferrara, 78; Viale Ferrero, Museo PoldiPezzoli, 1984, parle plus exactement de " una libera trascrizione imitativa ".

51 V. Hoffmann, "Leonardos Ausmalung der Sala delle Asse im Castello Sforzesco ", Mitteilungen des Kunsthistorischen Institutes in Florenz, xvI (1972), 56; " le lierre se mélange aux
Pour Ferrante Gonzaga, son commanditaire, cette tenture doit avoir symbolisé avant tout le thème de la Paix et des délices de la vie. Elle est ainsi complémentaire aux Fructus Belli, la tenture précédente qu'il fit tisser à Bruxelles en 1546-1547 et qui évoqua les désastres de la Guerre, thème qui prévalut durant sa carrière de généralissime des troupes impériales ${ }^{52}$. Gouverneur de Milan et de la Lombardie depuis 1546, il s'apprêta à jouir d'une vie un peu plus sédentaire, malgré la campagne du Piémont.

Ne peut-on dès lors pas reconnaître une allusion à ses lieux de séjour favoris dans les architectures si clairement rendues aux arrières-plans? $\mathrm{Si}$ les édifices majestueux de la Ville (Fig. 89) évoquent avant tout la cité idéale de la Renaissance, le palais à l'arrière droite de la Danse (Figs 94 et 95) offre des ressemblances assez frappantes avec l'aspect général de la villa Gualtiera-Simonetta, la résidence de Ferrante à Milan, transformée pour lui dès 1546 par Domenico Giunti et dont la disposition nous est connue entre autres par des gravures ultérieures (Fig. 96) ${ }^{53}$.

L'absence d'armoiries des Gonzague indique, à notre avis, que Ferrante destinait cette tenture à son usage privé. Ceci est confirmé par la particularité, citée plus haut, que les cartons de cette tenture furent récupérés par le commanditaire, à l'opposé de ceux des Fructus Belli, retissés ensuite à Bruxelles vers 1600 . Le lissier ne pouvait ainsi la remettre sur le métier et le cardinal de Granvelle devra racheter les cartons auprès des héritiers de Ferrante en 1573, soit vingt ans environ après le tissage de l'editio princeps.

Aussi au point de vue formel, la tenture des Jeux d'Enfants de Ferrante diffère considérablement de celle de son frère, le cardinal Ercole. Constatons d'emblée que la paternité de Giulio semble exclue dès l'origine pour cette série. L'artiste étant décédé en 1546, on s'imagine mal que Gian Battista Lodi apporte encore ses modéles à Willem de Pannemaker en février 1552. Ici, toute l'action est confiée aux enfants, à l'exclusion de tout autre personnage. Les enfants eux-mêmes sont d'un dessin plus lourd que ceux de Giulio, et ils ne sont pas munis d'ailes. Les pergolas des vignes dans les chênes témoignent elles aussi d'une lourdeur de dessin plus prononcée. Le feuillage des chênes est d'une conception nettement nordique, rappelant celui de la tenture bruxelloise contemporaine de la Genèse, achetée à Anvers par

arbres les plus hauts, tel que la vigne la plus noble ", selon Aelianus, Variae historiae, III-1, publiées dès 1545 à Rome. 52 Delmarcel, "Fructus Belli ".

53 A. Castellano, "La villa milanese nella prima metà del Cinquecento. Un prototipo inedito: la Gualtiera-Simonetta ", La Lombardia spagnola (Milano, 1984), 89, fig. 93. 
Cosimo I de'Medici en $1551^{54}$. La végétation de l'avant-plan, avec le plantain et d'autres mauvaises herbes, est commune elle aussi à la production bruxelloise de l'époque.

Les éléments généraux de la composition et du dessin sont sûrement d'origine italienne, et l'on peut supposer que, comme pour la tenture précédente des Fructus Belli, Ferrante aura envoyé des petits patrons italiens aux Pays-Bas, où ceux-ci furent agrandis en cartons, avec les adaptations et infidélités que cela peut comprendre par rapport au modèle original. Le maquettiste encore inconnu qui a fourni les petits patrons était peutêtre issu de la bottegha de Romano; l'attribution de ces tapisseries à Raphaël dans les inventaires du $\mathrm{XVIII}^{\mathrm{e}}$ siècle, cités plus haut, confirme peut-être une telle tradition. Ou doit-on penser à un peintre milanais de l'entourage de Ferrante, gouverneur de la ville de 1546 à 1554 ? Rappelons ici que des peintres cartonniers, travaillant à une série de "ispalliere ", sont cités au service de Ferrante à Milan dès mars $1547^{55}$. La conception des enfants mêmes, ainsi que les dessins d'architecture des Jeux d'Enfants, sont d'une veine toute italienne. Une influence sinon une conception milanaise de cette tenture pourrait être déduite de la structure très prononcée des tonnelles en forme de pergola, qui n'est pas sans rappeler les fresques de la fameuse Sala delle Asse, peintes au Castello Sforzesco par Leonardo da Vinci en 1495-1498. Là aussi, les frondaisons se mêlent; elles y sont reliées par des noeuds et des liens. Bien que l'iconographie de cette salle trouve peut-être son origine dans une évocation funéraire de Béatrice d'Este, elle peut aussi évoquer le thème commun de la vallée de Tempe ${ }^{56}$.

Le thème dionysiaque de la tenture est repris dans le dessin des bordures. Entre deux minces frises d'acanthe, des têtes de satyres et bacchantes et des gueules de lion sont reliées par des pampres et des grappes de raisin. Dans les bordures verticales, les masques tiennent ces pampres par des noeuds entre les dents. Les satyres ne sont pas sans ressemblance avec une tête similaire, dans la bordure de droite du Passage de la Mer Rouge, une des pièces de la tenture de Moïse aux armoiries du duc Giuglielmo Gonzaga, le successeur de Federico. Cette tenture, qui fut offerte par la suite au

54 Florence, Accademia, cf. R. A. d'Hulst, Tapisseries flamandes (Bruxelles, 1960), 231-38; Adelson, "Cosimo ", 915.

55 C. Baroni, Documenti per la storia dell'Architettura a Milano nel Rinascimento e nel Barocco (Rme, 1968), I, 483 (lettre de l'architecte Guinti à Ferrante Gonzaga).

56 Hoffmann, "Leonardos Ausmalung ", 51-62; M. G. Agghàzy, "Locus amoenus et vinculum delictorum dans l'art de la Renaissance ", Bull. du Musée hongrois des Beaux-Arts, LI (1978), 55-62.
Duomo de Milan par saint Charles Borromée, aurait été conçue elle aussi par des épigones de Giulio Romano et elle aurait été tissée par Nicolas Karcher lors de son second séjour à Mantoue, dans les années 1555-1562, donc à la même époque que les Puttini de Ferrante ${ }^{57}$.

Les Jeux d'Enfants des deux frères Gonzague, le cardinal Ercole et le général Ferrante forment deux ensembles apparentés, conçus l'un par le grand "inventor " de Mantoue et l'autre par des artistes italiens dans sa foulée, et tissés par des lissiers nordiques, l'un peut-être à Mantoue même, l'autre à Bruxelles. Ces tapisseries témoignent de l'intérêt de leurs commanditaires pour les thèmes antiques de la vie paradisiaque, reliés probablement à une symbolique partiellement chrétienne. Si notre contribution aura précisé quelque peu leur histoire et leur sens, tous leurs secrets ne sont pas encore révélés, ce qui n'ôte rien à leur charme.

\section{DOCUMENTS}

I

Lierre, 5 février 1552 : lettre du peintre Gian Battista Lodi à Ferrante Gonzaga concernant le tissage d'une tenture de tapisserie en fils d'or et de soie à Bruxelles.

All'Illustrissimo et Eccellentissimo Signore il Signor Don Ferando Gonzaga, Capitan Generale di Sua Maestà et Governatore del Stato di Milano et cetera, Signor mio osservandissimo.

Illustrissimo et Eccellentissimo Signor mio Signor Osservandissimo,

Hauta la commissione di Vostra Eccellentia sopra le tapezzarie da farse subito, il Nicola et io andassimo a Brusselles, dove fatta ogni diligenza, al fine habbiamo fra tutti gli altri trovato uno sufficiente con molti et buoni lavoranti, accomodato di fine sete d'ogni colore, il quale per la Cesarea Maestà riduce in tapezzaria d'oro l'impresa di Tunis. Gli mostriammo l'opera con buon modo. Egli domanda per l'ultimo prezzo, a farle ricche d'oro et di finissime sete, quatuordeci fiorini per ala. $\mathrm{E}$ con che ci assottigliassimo assai, non si puotè tirar a manco. Ma volendoli dar noi l'oro, le farà, per otto fiorini l'ala, di sete finissime, e l'oro gli daremmo promette lealmente et bene metter ne l'opera.

Egli ha fama del miglior mastro che ivi si trove, et per tale l'habbiamo riputato noi a le opere sue. Se sarà il nobil piacere di Vostra Eccellentia farle espedire per lui, la suplico humilmente farne avertire qual de duo modi più le agrada.

Non mi pare se gli debba mettere argento, perché in breve divien negro et disdice assai. Nientedimanco la volontade di Vostra Eccellentia sia fatta a la cui, come buono et humil servitore che le sono, inchinevole mi rimetto per sempre. Et pregando Idio per la felicitade di Vostra Eccellentia le bascio con tutta riverenza le illustrissime mani.

57 Forti, Ferrara, 79 et 197, pl. 144. 
Da Lira a li v di febraro mDLr ab incarnatione. Di Vostra Eccellentia

Hurnilissimo servitore - Battista Lodi pittore

(Aspr, Epistolario Scelto, Busta 18, Publ. par J. A. F. Orbaan, "Parmezaansche gegevens", Oud Holland, xLI [1923-1924], 252-53)

Avant avril 1587: Guglielmo Gonzaga, duc de Mantoue, envoie le juif Modone à Venise pour essayer d'y acheter une pièce des Jeux d'Enfants des Gonzague de Guastalla.

Instructione a Modone hebreo di quanto ha da fare a Venezia per servigio di Sua Altezza.

Primeramente ha da vedere se la tapezzaria che ha colla l'Eccellentissimo Signor Don Ferrante Gonzaga, chiamata delli Puttini, si confa con quella di Sua Altezza che chiamano del medesimo nome, della quale n'havrà un pezzo da portar seco et trovandola tale et che sia in tanta quantità che basti ad apparare la camera, della quale li sarà data la misura dal signor Giovanni Ceresara, procurerà con quelli migliori mezzi che saprà di tirarla al più basso et avantagioso prezzo che si possa per servigio di Sua Altezza. Et quando havrà inteso l'ultimo prezzo, procurerà di farsene dar un pezzo da portar seco a far vedere a Sua Altezza accio ch'ella possa rissolvere se le piacerà la tapezzaria et il mercato. [...]

(ASMn, Busta 1417)

\section{APPENDICE}

Après avoir terminé la rédaction de cet article, une découverte ultérieure nous permet de préciser actuellement la date de l' "Instructione a Modone " (ASMn, Busta 1417-voir notre document II). Un autre volume des archives Gonzaga (Asmn, Busta 2953, Copialettere, libro 385) contient des copies de lettres écrites par le duc Guglielmo en 1579. L'une d'elles (carta 75v du 6 octobre 1579) est celle de Paolo Moro concernant la commission du dernier groupe de quatre peintures de Tintoretto, destinées à décorer les appartements derrière la Sala di Manto dans le nouveau complexe du Corte Nuovo ${ }^{58}$. Plusieurs pages auparavant se trouvent des lettres adressées à Giovanni Ceresara et à Giovanni Carlo del Re (carte $74 \mathrm{r}^{0}$ et $\mathrm{v}^{0}, 4$ octobre 1579), toutes deux à propos de l'arrivée à Venise de "Modone hebreo ". Dans la lettre à Ceresara, Guglielmo fait spécifier :

il signor nostro serenissimo manda Modone hebreo a Vinegia per haver certe tapezzarie per guarnire la camera nuova che è oltre quella dell'Aquila. Perciò Vostra Signoria sia contenta di far prendere la misura delli

58 P. Eikemeier, "Der Gonzaga-Zyklus des Tintoretto in der Alte Pinakothek ", Münchner Jahrbuch der bildenden Kunst, xx (1969), 75 et ss. pezzi che vanno intorno alla detta camera et di darla ad esso Modone.

Les instructions à Modone, citées dans notre document II, se rapportent ainsi sans aucun doute à ce voyage en octobre 1579. La date précise de cet événement a une signification plus qu'académique. A cette époque, le duc Guglielmo s'occupait activement de redéfinir des parties du palais ducal de Mantoue. Pour les appartements les plus récents, ceux du Corte Nuovo, l'on demanda la collaboration active de Tintoretto ${ }^{59}$. Avant d'ériger le Corte Nuovo, on travailla aux appartements privés de Guglielmo à la Corte Vecchia (l'actuel Appartamento degli Arazzi), pour lesquels on fit appel aux talents de Pirro Ligorio ${ }^{60}$. La plupart des travaux exécutés pour Guglielmo dans la Corte Vecchia ont disparu. C'était là que se trouvait la "camera nuova che è oltre quella dell'Aquila [Camera del Leone?] ". Et quand les appartements de cette partie du palais furent effectivement ornés de tapisseries en octobre 1580 , ce sont les Puttini du cardinal Ercole qui furent placés dans cette même "stantia dell" Aquila "(ASMn, Busta 2210 : Federico Donato6 octobre 1580).

$\mathrm{Vu}$ que les appartements en question n'existent plus, il n'est plus possible de les comparer aux dimensions des Puttini d'Ercole (dont on ne possède, en tout cas, qu'une partie) et de se demander par conséquent si la requête de Guglielmo, ou du moins son intention, ne se rapportait simplement à un prêt temporaire d'un ou de plusieurs panneaux de la tenture de Ferrante afin de compléter la couverture des surfaces murales. C'est une alternative valable pour la supposition que la tapisserie que Modone devait ramener à Mantoua, aurait dû servir comme modèle pour remplacer ou réparer une tapisserie endommagée. Nous savons que les Puttini d'Ercole furent sortis de la réserve en 1584 en l'honneur de la nouvelle épouse de Vincenzo Gonzaga. Nous ignorons jusqu'à présent si leur déploiement en octobre 1580 fut motivé par quelque autre circonstance particulière.

\section{Musées royaux d'Art et d'Histoire Parc du Cinquantenaire, 10 B 1040 Bruxelles Carleton University Ottawa, Ontario K1S 5B6}

59 C. Tellini Perina, "Considerazioni su alcuni aspetti della cultura figurativa del Cinquecento a Mantova ", Antichità Viva, xill (1974), 17 et ss.

60 C. Cottafavi, "Ricerche e documenti sulla costruzione del Palazzo Ducale ", Atti e Memorie dell'Accademia Virgiliana, n.s. $x \times v$ (1939), 203 et ss. 


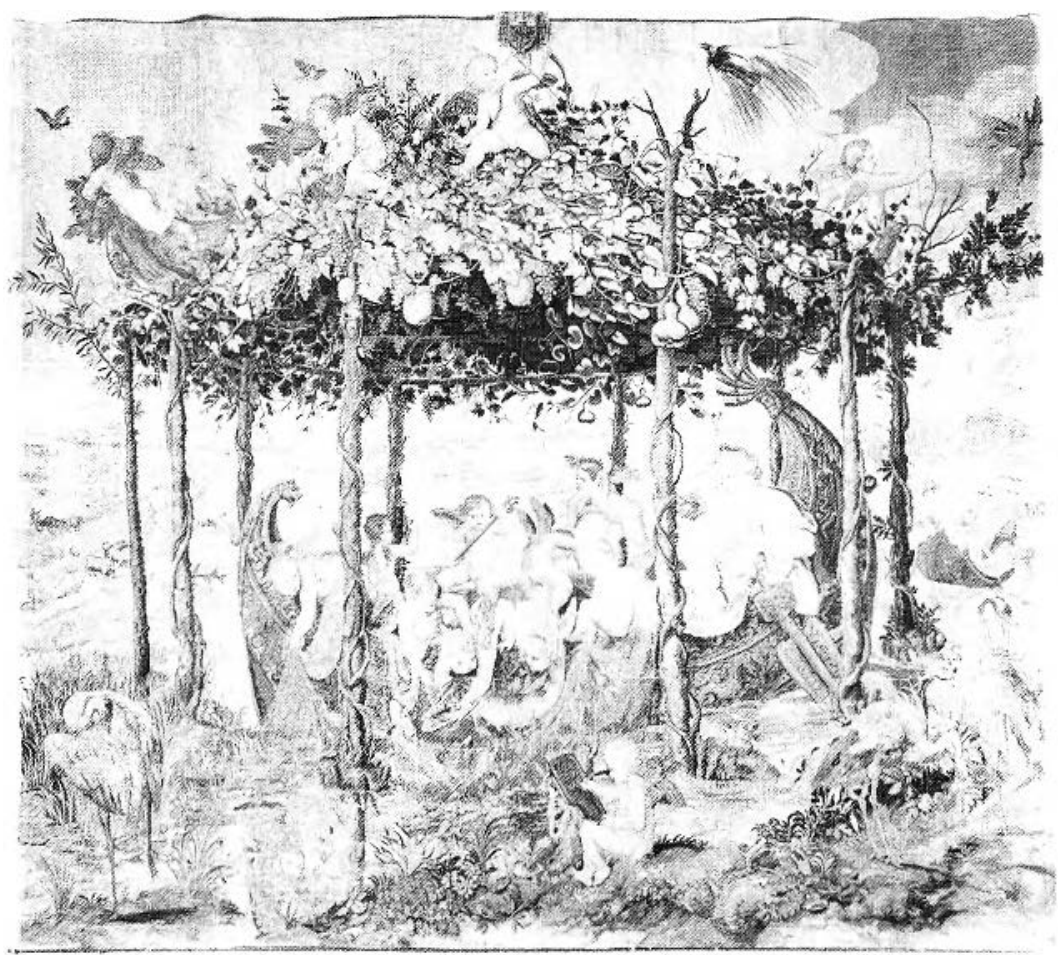

Figlere 79. La Barque de Vénus/Fortune. Tapisserie italienne d'après Giulio Romano. Atelier de Nicolas Karcher, Mantoue (?), vers 1539-1545. Musće Gulbenkian, Lisbonne (Photo: Musée Gulbenkian)

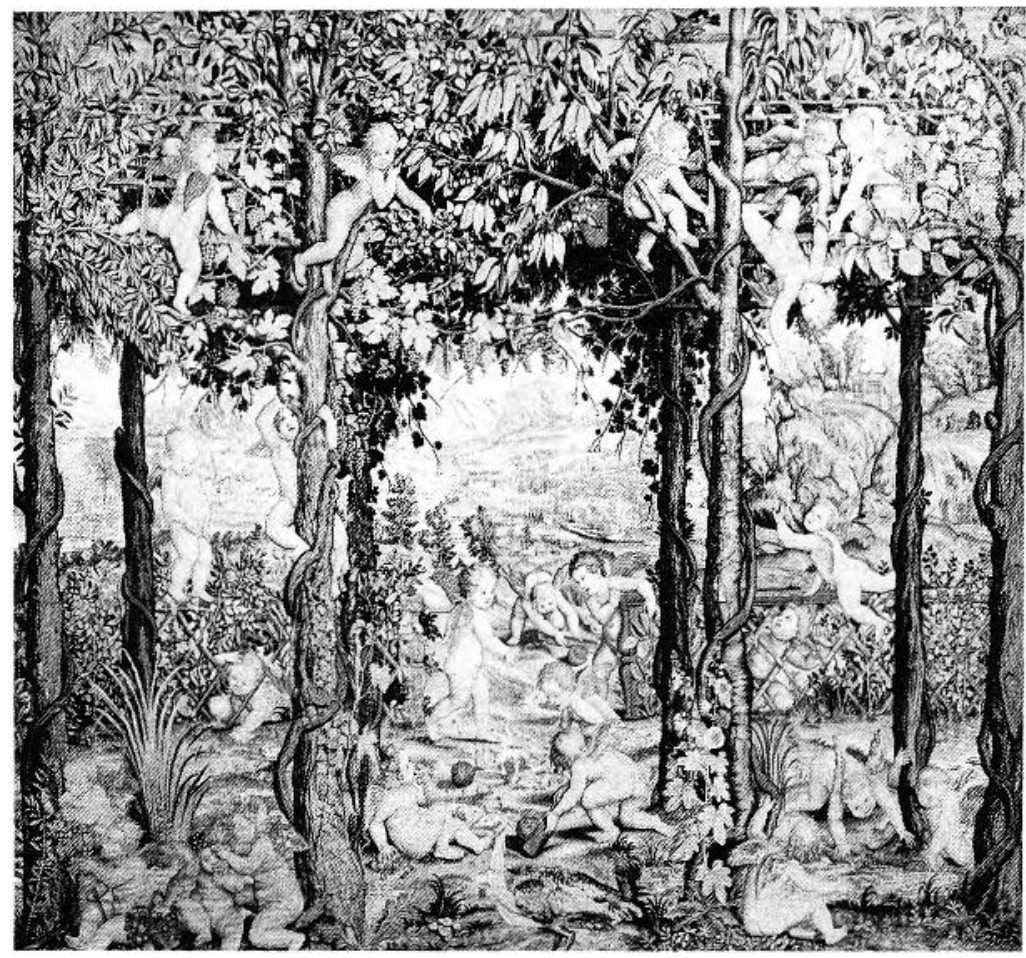

Figure 80. Le Jeu de Balle. Musée Gulbenkian, Lisbonne (Photo: Musée Gulbenkian). 


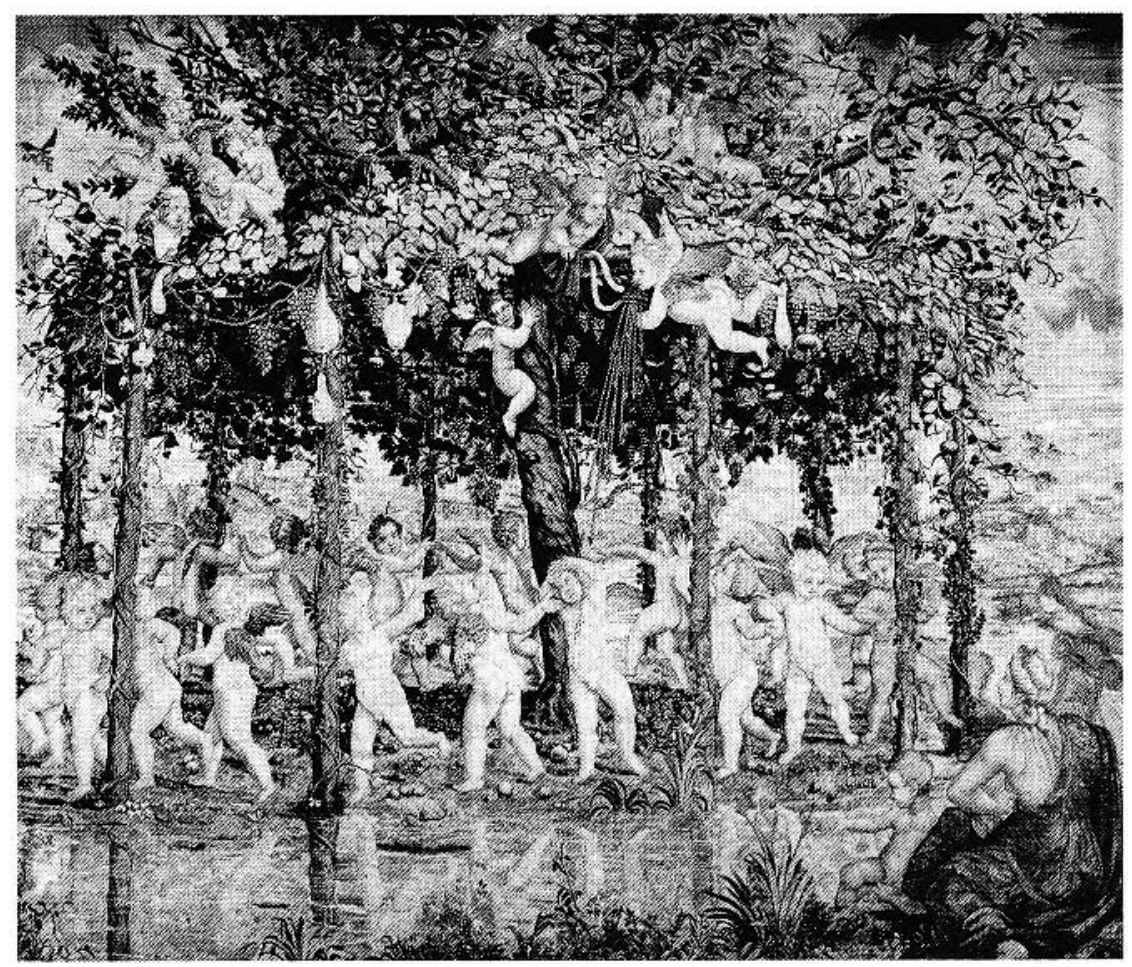

Figure 81. La Danse. Musée Gulbenkian, Lisbonnc (Photo: Musée Gulbenkian).

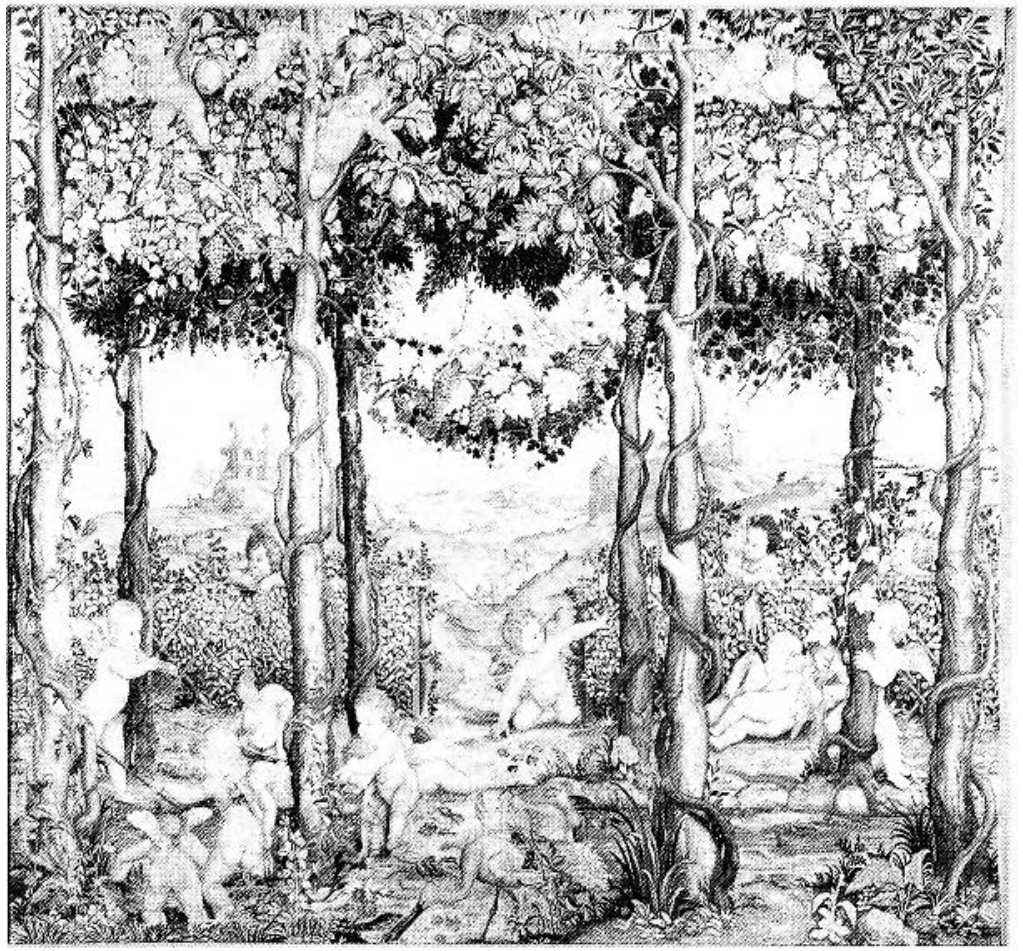

Figure 82. La Pêche. Musée Gulbenkian, Lisbonne (Photo : Musée Gulbenkian). 


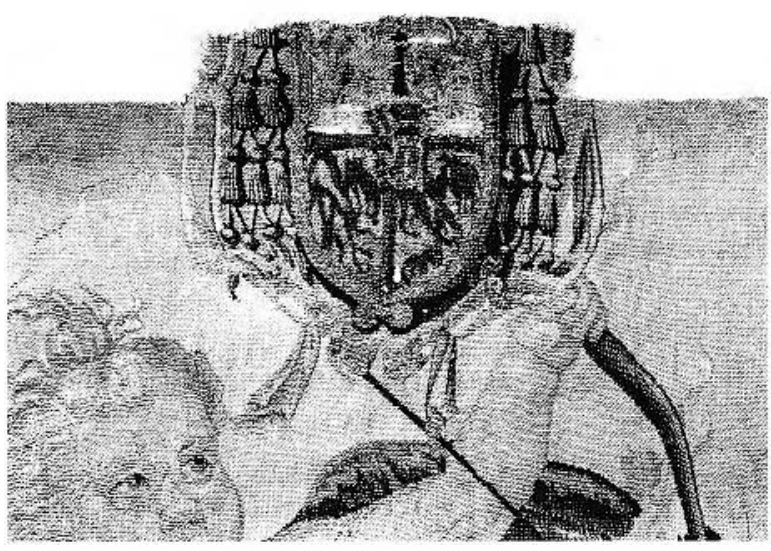

Figure 83. Détail de la Fig. 79 : armoiries du cardinal Ercole Gonzaga (Photo: Musée Gulbenkian).

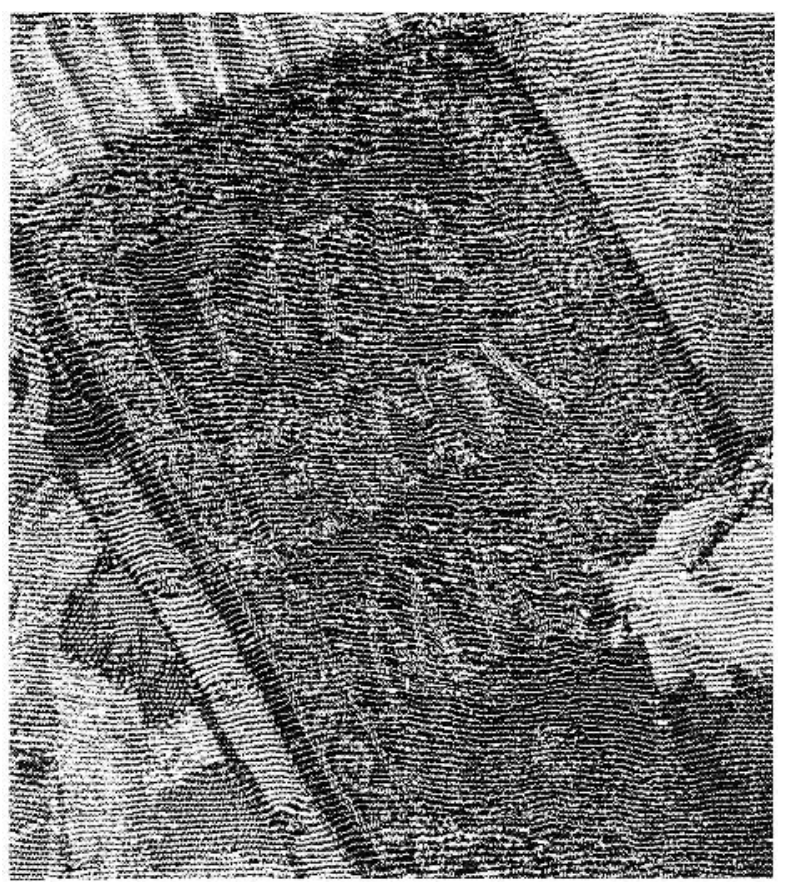

Figure 84. Détail de la Fig. 79: inscription à l'avantplan (Photo: Musée Gulbenkian).

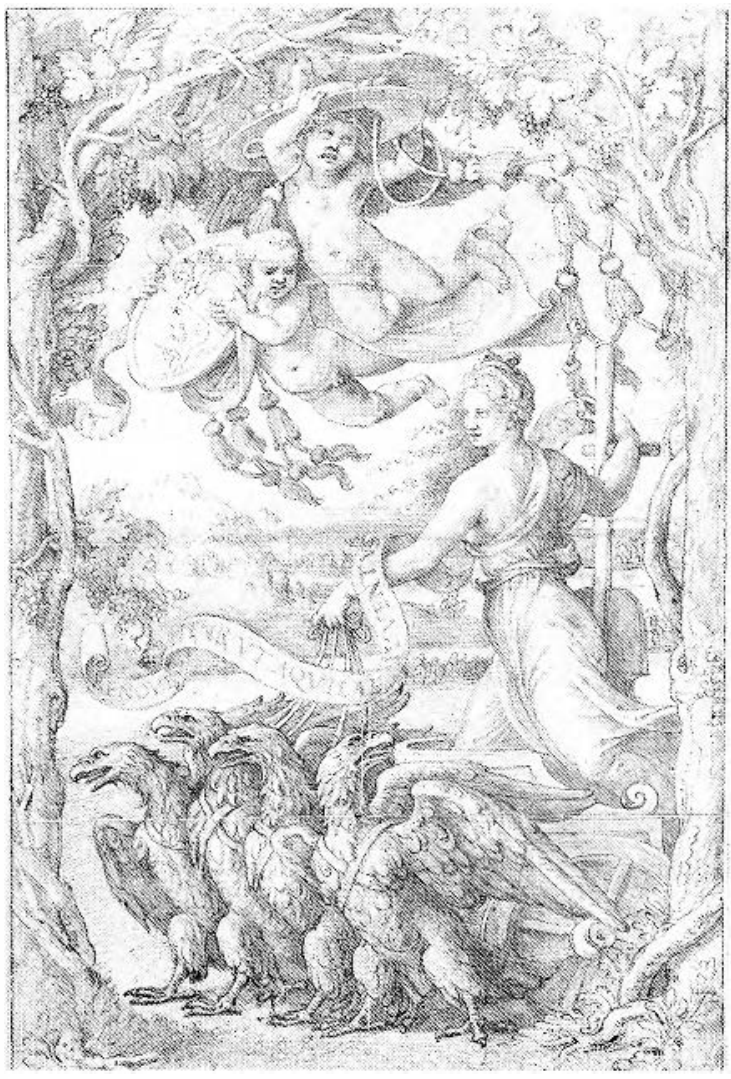

Figure 85. (Giulio Romano, La Fortune menée par les aigles des Gonzague, dessin. Teyler Museum. Haarlem (Photo: Teyler Museum).

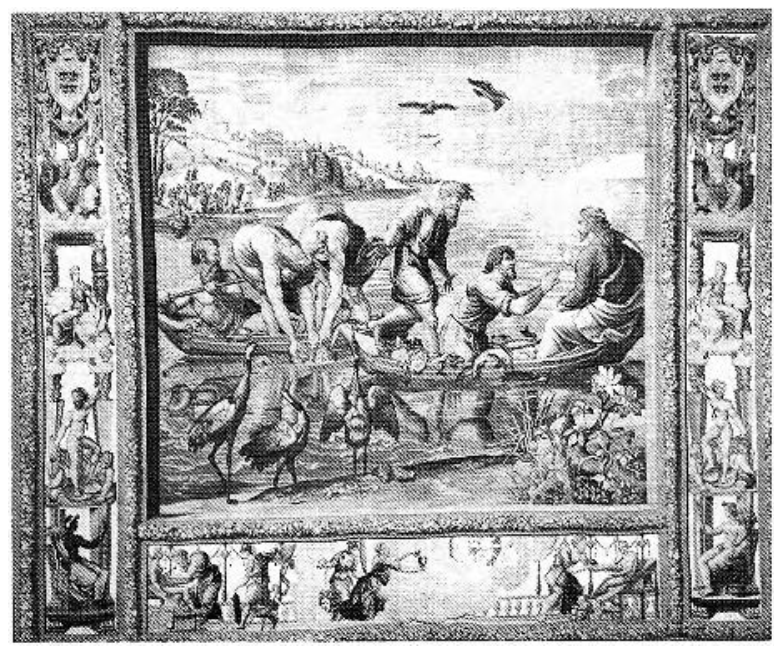

Figure 86. La P’êche Miraculeuse, tapisseric bruxelloise aux armes du cardinal Ercole Gonzaga (après restauration). Atelier de Jan Van Tieghcm, Biuxelles, 1562-1563. Palazzo) Ducale, Mantoue (Photo: A.c...., Bruxelles). 


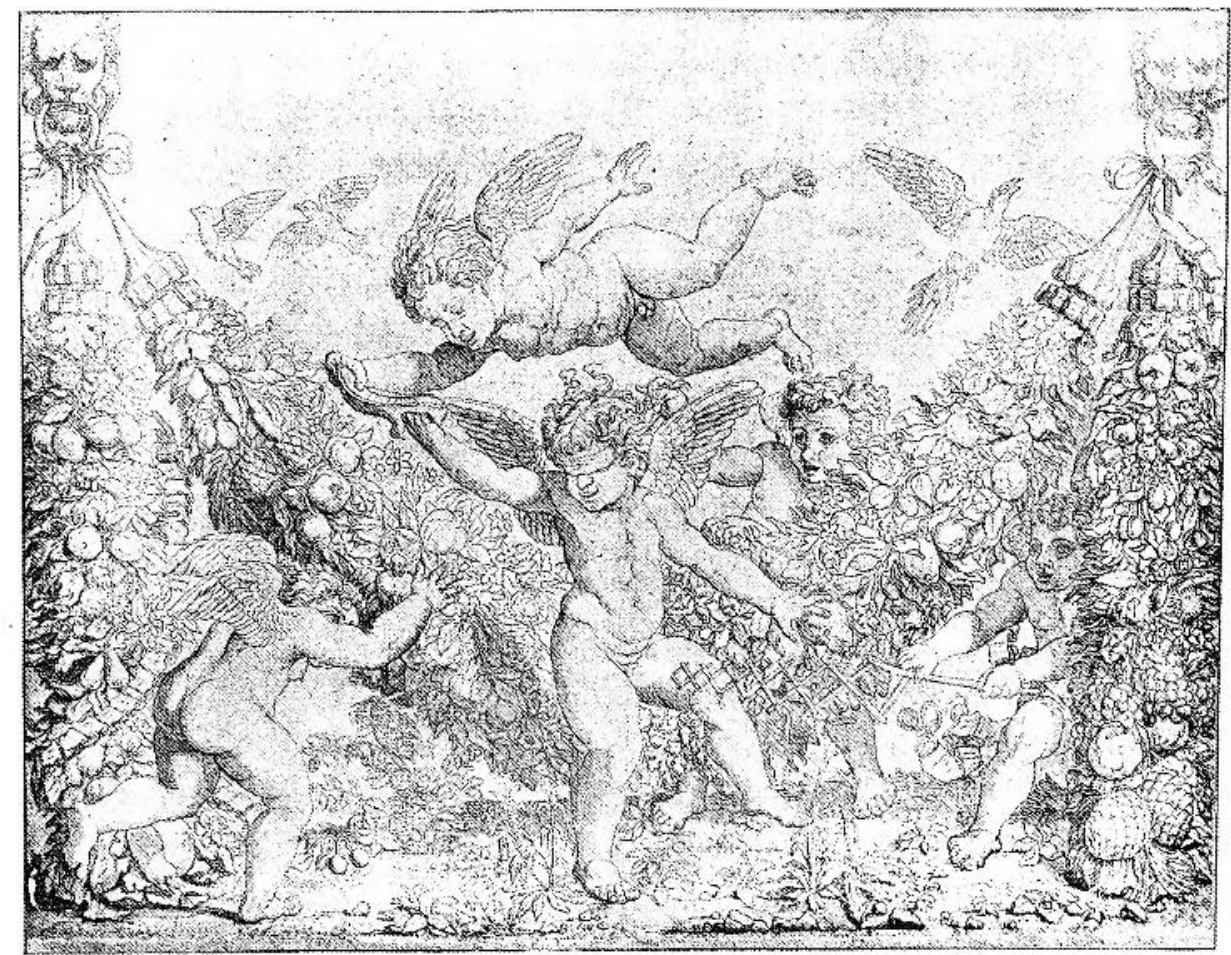

Figure 87. Dessin de Giovanni da Udine, les Jeux d'Fnfants pour Léon x. Lieu de conservation actucl inconnu, d'après E. Münt\% (Photo: P. Stuyven, Louvain).

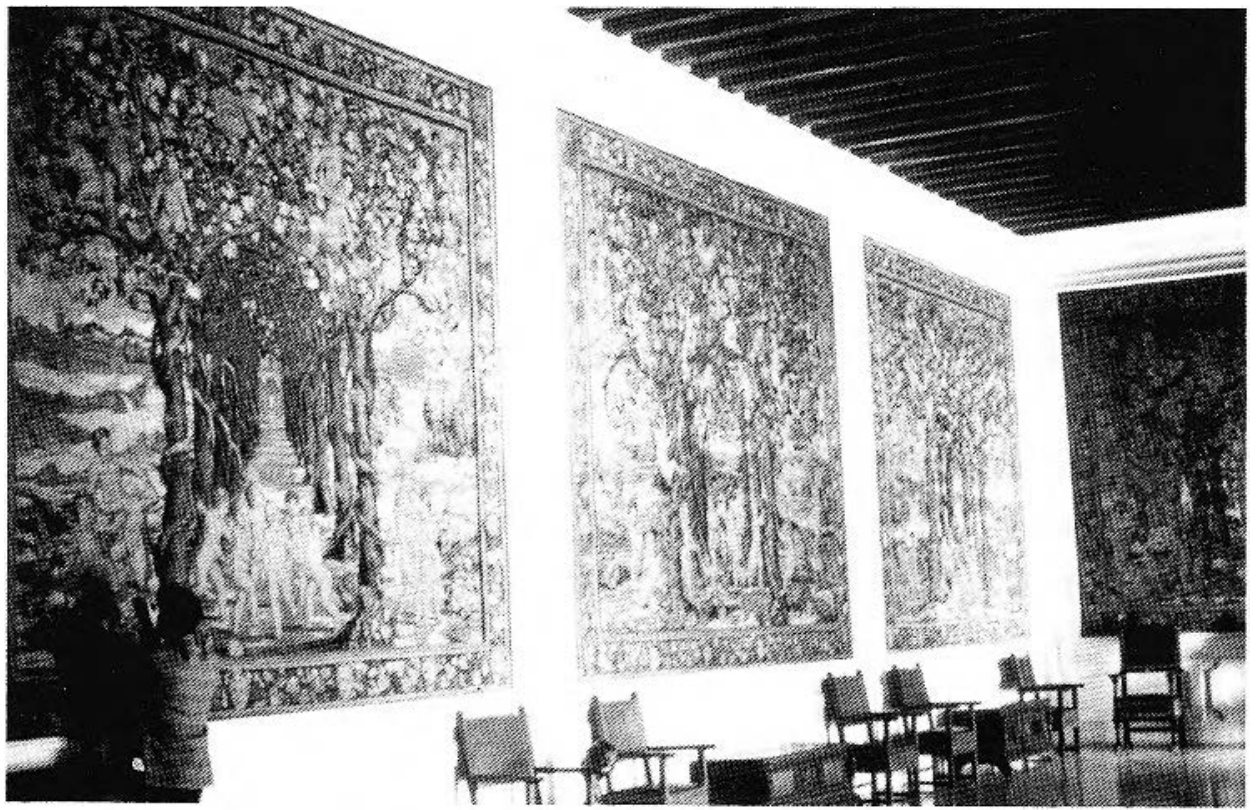

Figure 88. La tenture des Jeux d'Enfants de Ferrante Gonzaga, vue générale. Collection Comte Giannino Marzotto, Villa 'Irissino, Trissino (Photo: C. Brown). 


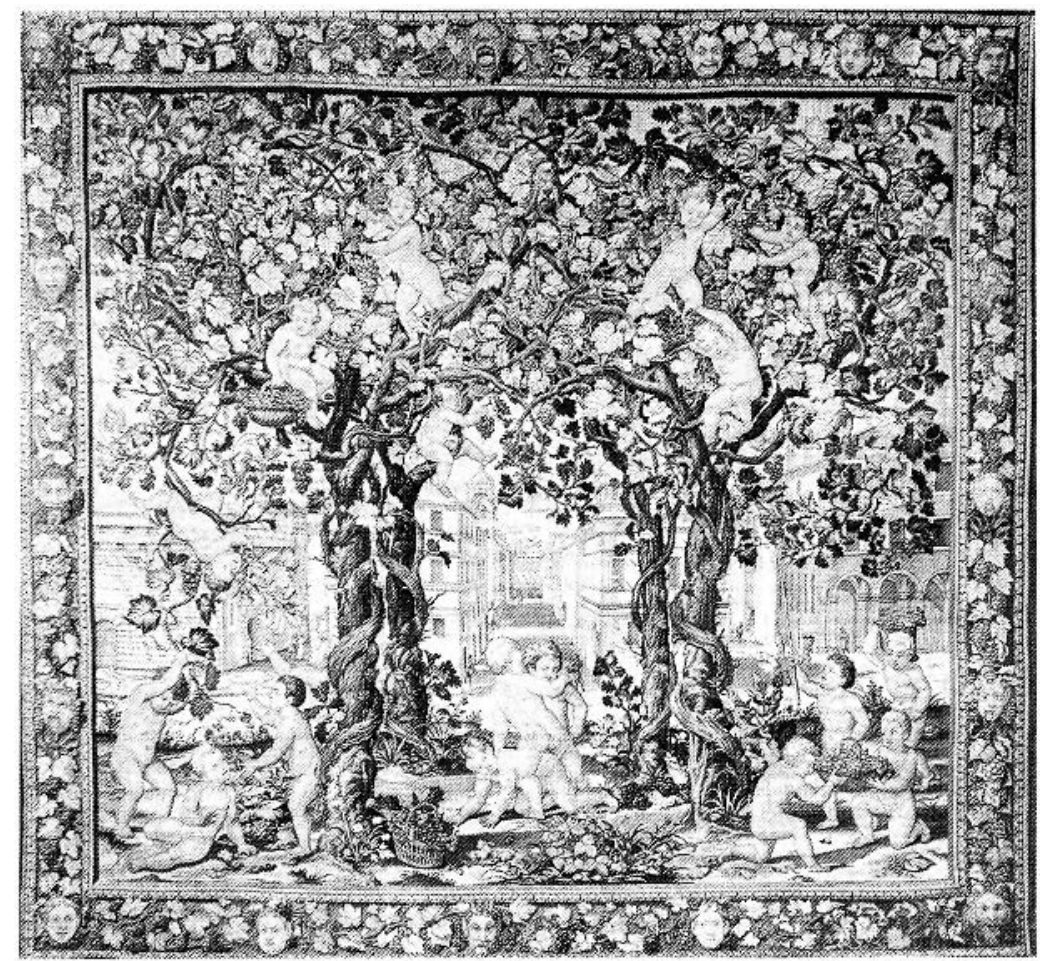

Fiscure 89. La Ville. Tapisscrie bruxelloise, atelier de Willem de Pannemaker, 1552-1557. Coll. Marzotto, Trissino (Photo : Giovetti, Mantoue).

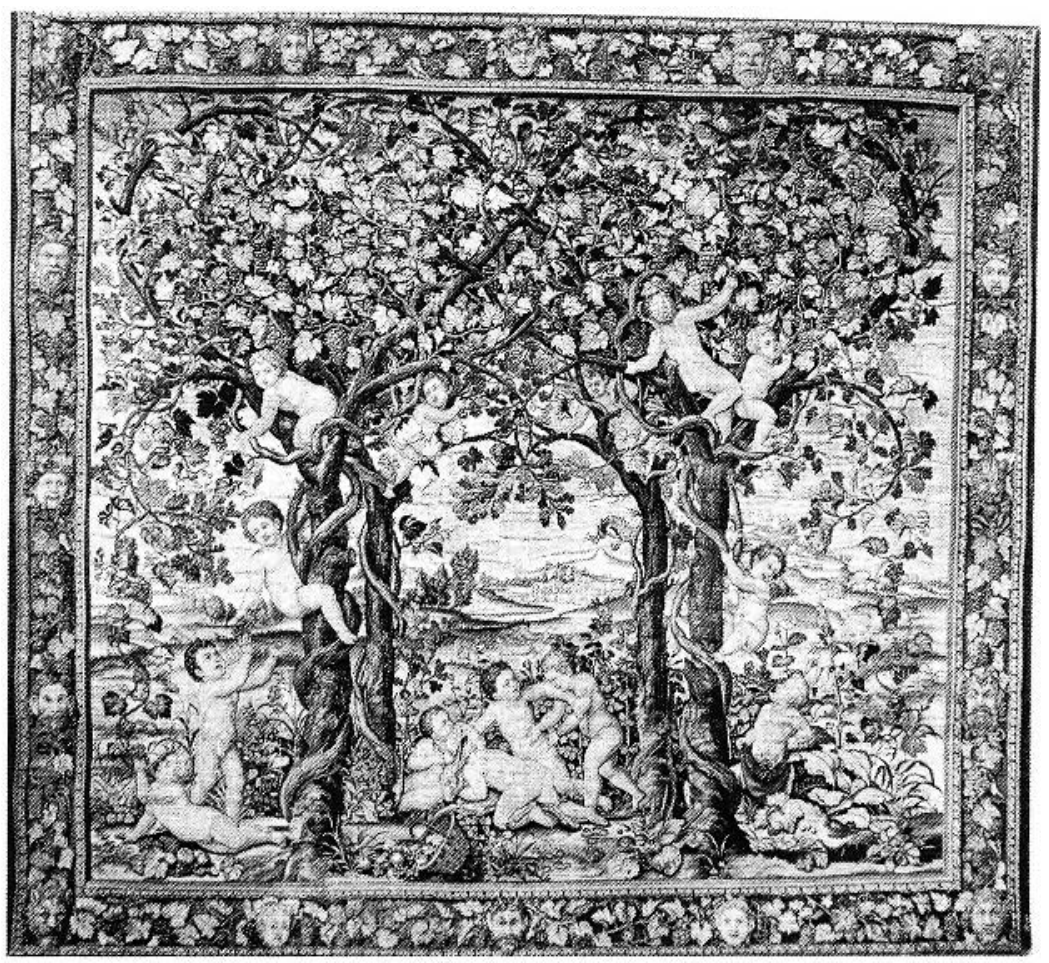

Figure. 90. Le Lièvre. Coll. Marzotto, Trissino (Photo: Giovetti, Mantoue). 


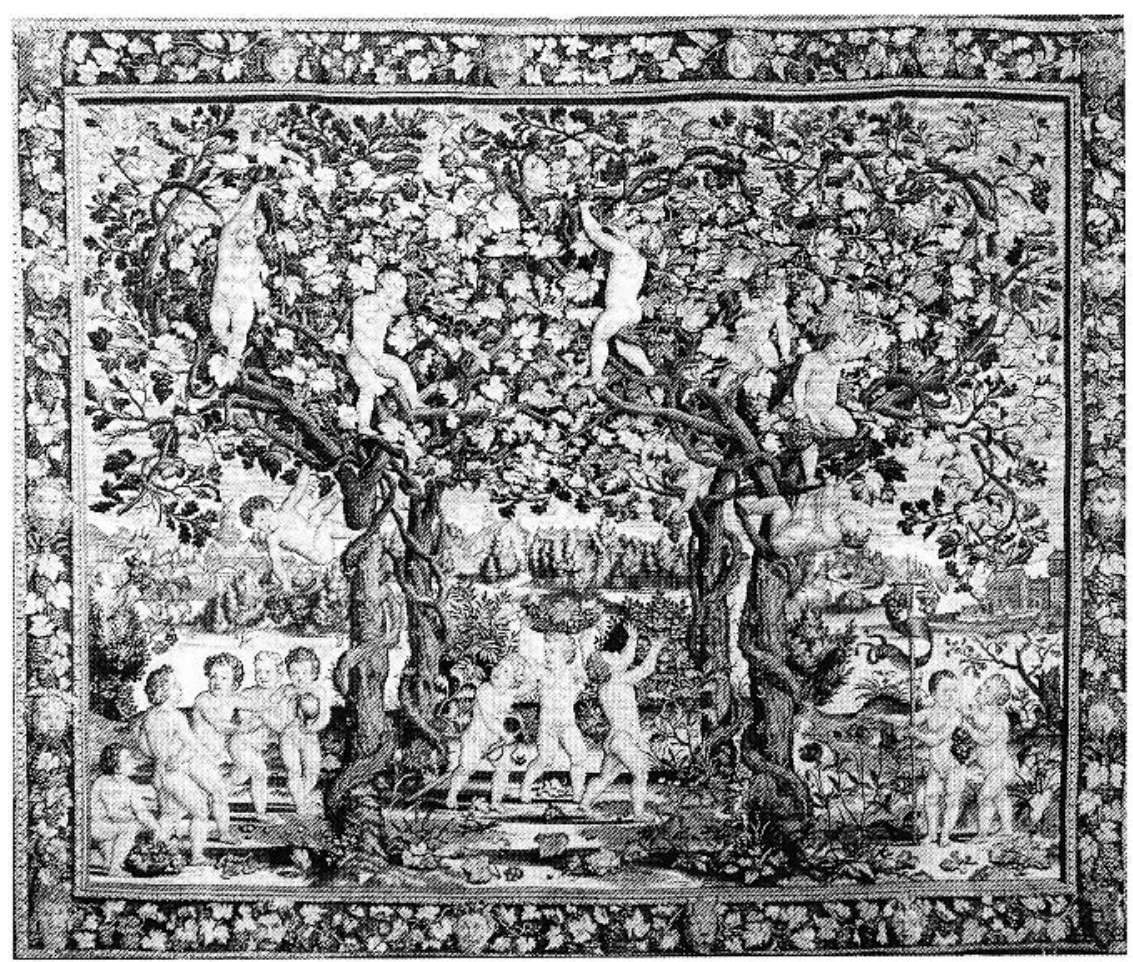

Figure 91. Lc Cheval-Bâton. Coll. Marzotto, Trissino (Photo: Giovetti, Mantoue).

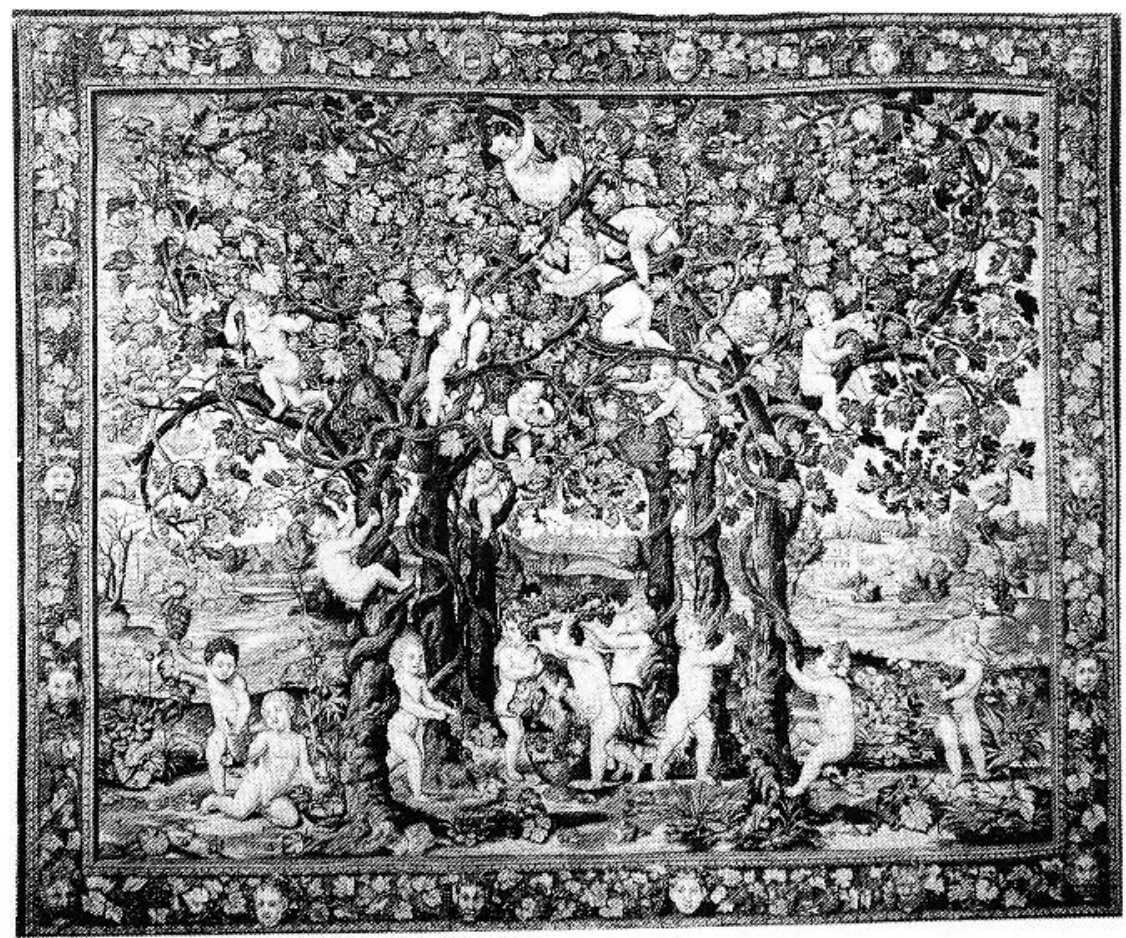

Figure 92. Le Bouc. Coll. Marzotto, Trissino (Photo: Giovetti, Mantoue). 


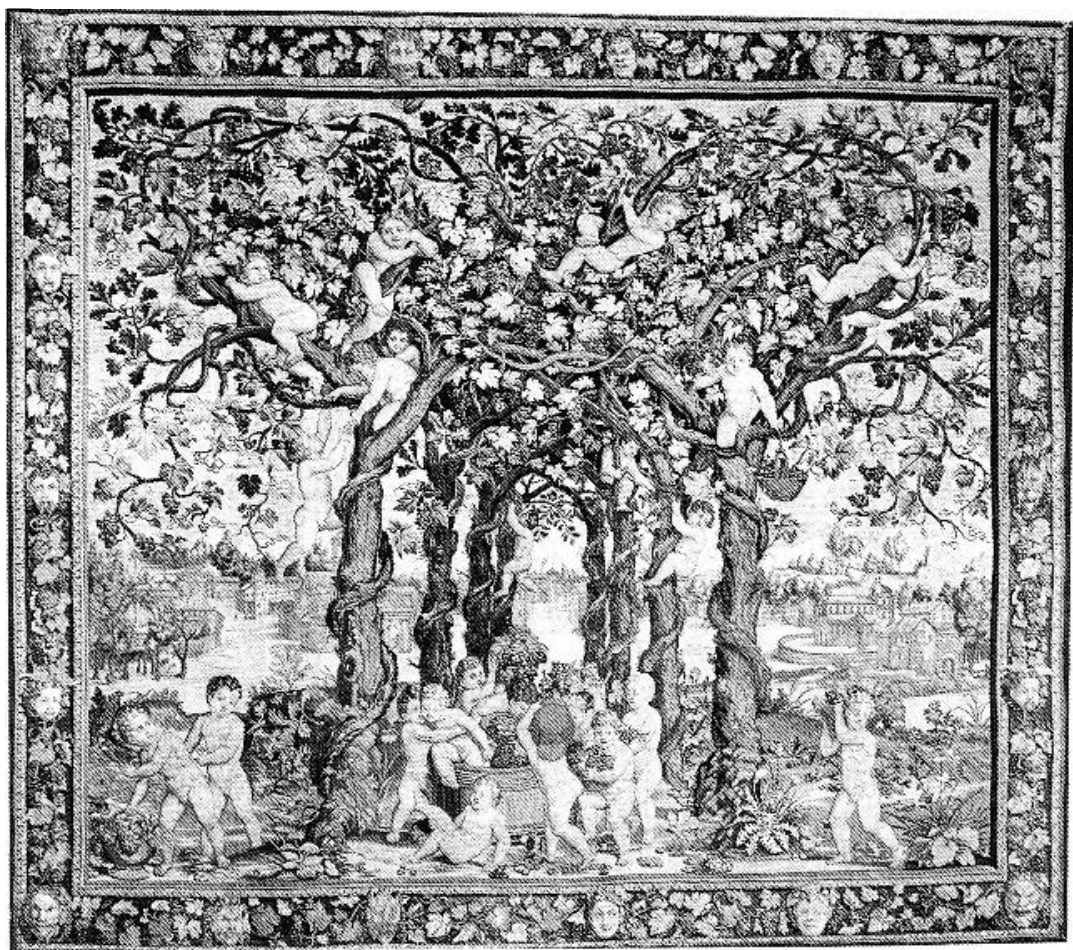

Ficivre 93. Le Pressoir. Coll. Marzotto, Trissino (Photo: (Giovetti, Mantoue).

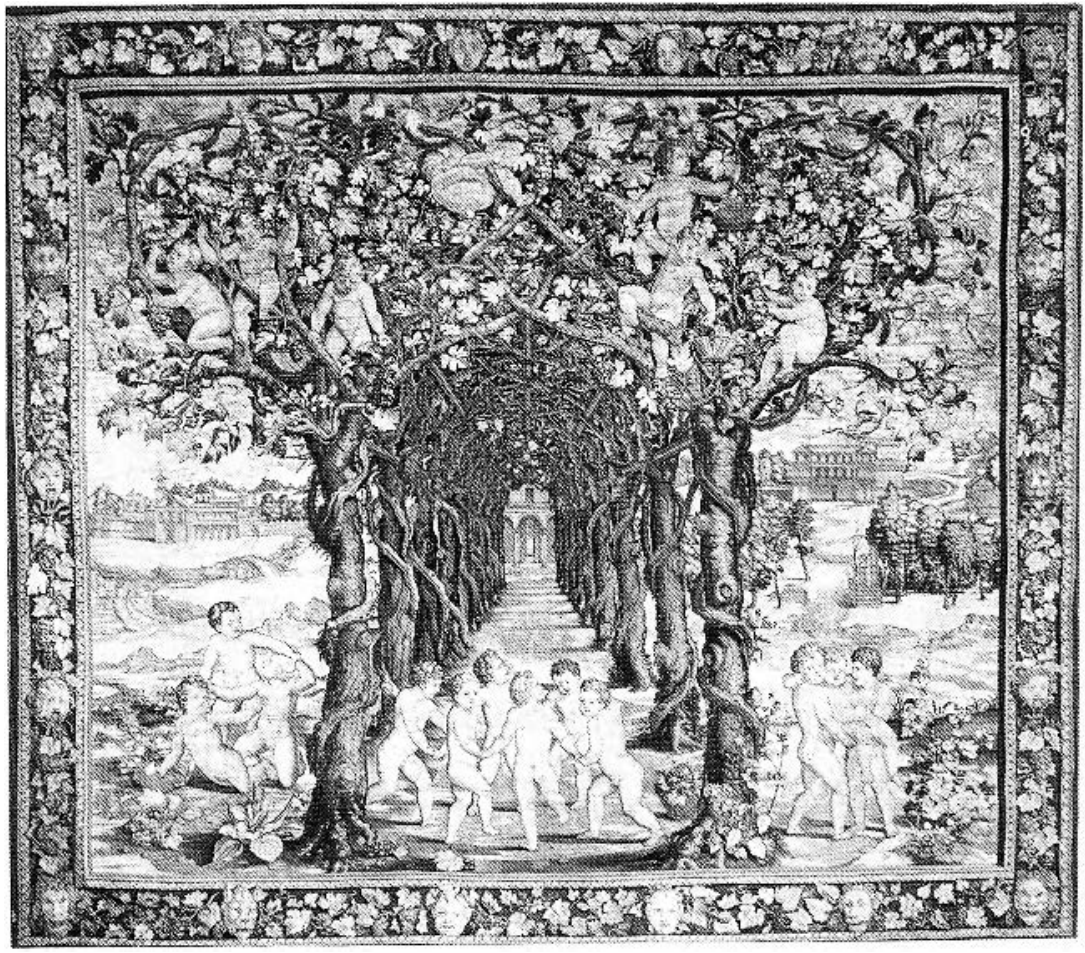

Figepe 94. i a Danse. Coll. Marzotto, Trissino (Photo: Giovetti, Mantouc) 


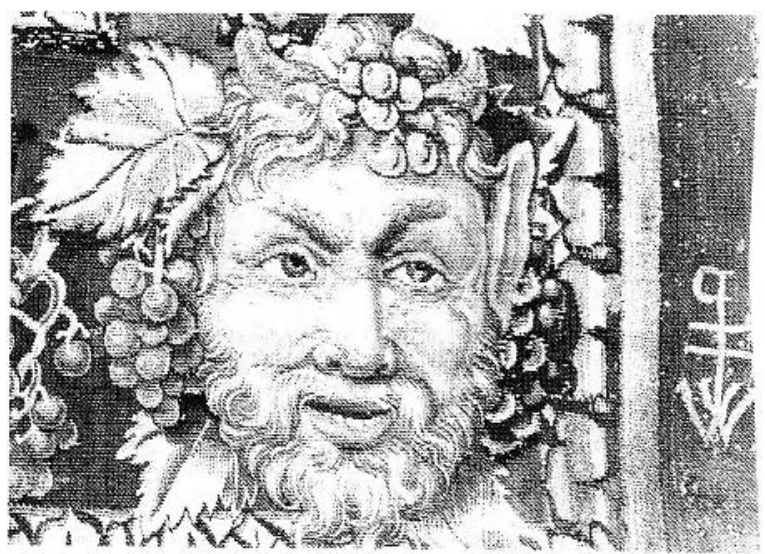

Ficiure 97. Détail de la Fig. 92 : marque de Willem de Pannemaker (Photo): G. Delmarcel).

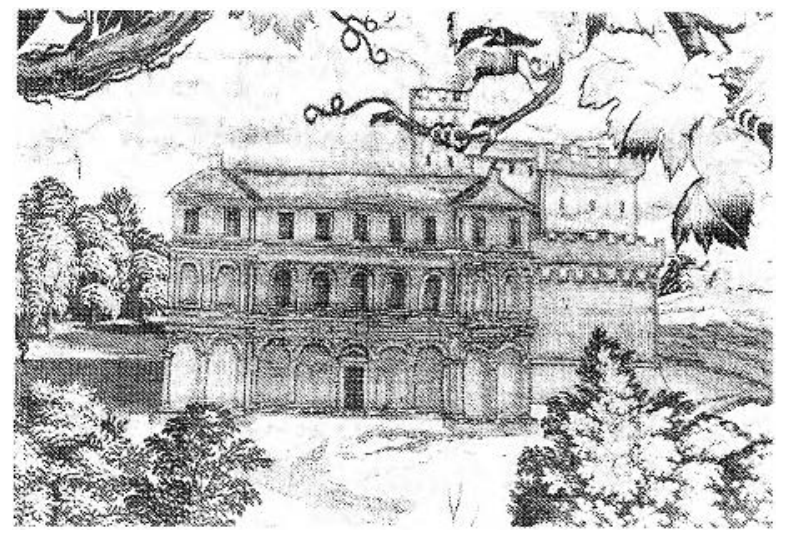

Figure. 95. Détail de la Fig. 94 : ćdifice à l'arrièreplan de droite (Photo: (;. Delinarcel).

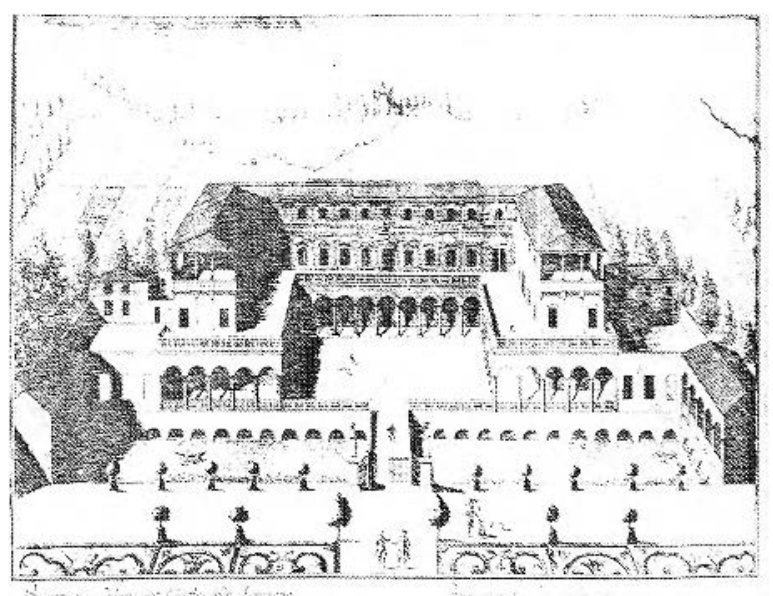

ligurf: 96. La Villa Gualtiera-Simonetta, d'après Marc Antonio dal'Re (lans Ville di delizia o siano palagi caparecci nello Stato di Milano, Milan 1726 (Photo: G. Delmarcel). 